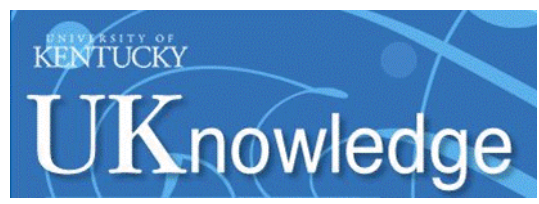

University of Kentucky

UKnowledge

\title{
Locally Optimally Emitting Clouds and the Narrow Emission Lines in Seyfert Galaxies
}

\author{
Jason W. Ferguson \\ University of Kentucky \\ Kirk T. Korista \\ University of Kentucky \\ Jack A. Baldwin \\ Cerro Tololo Inter-American Observatory, Chile \\ Gary J. Ferland \\ University of Kentucky, gary@uky.edu
}

Follow this and additional works at: https://uknowledge.uky.edu/physastron_facpub

Part of the Astrophysics and Astronomy Commons, and the Physics Commons

Right click to open a feedback form in a new tab to let us know how this document benefits you.

\section{Repository Citation}

Ferguson, Jason W.; Korista, Kirk T.; Baldwin, Jack A.; and Ferland, Gary J., "Locally Optimally Emitting Clouds and the Narrow Emission Lines in Seyfert Galaxies" (1997). Physics and Astronomy Faculty Publications. 121.

https://uknowledge.uky.edu/physastron_facpub/121

This Article is brought to you for free and open access by the Physics and Astronomy at UKnowledge. It has been accepted for inclusion in Physics and Astronomy Faculty Publications by an authorized administrator of UKnowledge. For more information, please contact UKnowledge@lsv.uky.edu. 


\section{Locally Optimally Emitting Clouds and the Narrow Emission Lines in Seyfert Galaxies}

Digital Object Identifier (DOI)

http://dx.doi.org/10.1086/304611

Notes/Citation Information

Published in The Astrophysical Journal, v. 487, no. 1, p. 122-141.

(C) 1997. The American Astronomical Society. All rights reserved. Printed in the U.S.A.

The copyright holder has granted permission for posting the article here.

This article is available at UKnowledge: https://uknowledge.uky.edu/physastron_facpub/121 
The Astrophysical Journal, 487:122-141, 1997 September 20

(C) 1997. The American Astronomical Society. All rights reserved. Printed in U.S.A.

\title{
LOCALLY OPTIMALLY EMITTING CLOUDS AND THE NARROW EMISSION LINES IN SEYFERT GALAXIES
}

\author{
JASON W. FERGUSON AND KIRK T. Korista \\ Department of Physics and Astronomy, University of Kentucky, Lexington, KY 40506 \\ JACK A. BALDWIN \\ Cerro Tololo Interamerican Observatory, ${ }^{1}$ Casilla 603, La Serena, Chile \\ AND \\ GARY J. FERLAND \\ Department of Physics and Astronomy, University of Kentucky, Lexington, KY 40506 \\ Received 1997 April 7; accepted 1997 May 1
}

\begin{abstract}
The narrow emission line spectra of active galactic nuclei are not accurately described by simple photoionization models of single clouds. Recent Hubble Space Telescope images of Seyfert 2 galaxies show that these objects are rich with ionization cones, knots, filaments, and strands of ionized gas. Here we extend to the narrow-line region the "locally optimally emitting cloud" (LOC) model, in which the observed spectra are predominantly determined by powerful selection effects. We present a large grid of photoionization models covering a wide range of physical conditions and show the optimal conditions for producing many of the strongest emission lines. We show that the integrated narrow-line spectrum can be predicted by an integration of an ensemble of clouds, and we present these results in the form of diagnostic line ratio diagrams making comparisons with observations. We also predict key diagnostic line ratios as a function of distance from the ionizing source and compare these with observations. The predicted radial dependence of the $[\mathrm{O} \mathrm{III}] /[\mathrm{O} \mathrm{II}]$ ratio may be matched to the observed one in NGC 4151, if the narrow-line clouds see a more intense continuum than we see. The LOC scenario when coupled with a simple Keplerian gravitational velocity field will quite naturally predict the observed line width versus critical density relationship. The influence of dust within the ionized portion of the clouds is discussed, and we show that the more neutral gas is likely to be dusty, although a high-ionization dustfree region is most likely present too. This argues for a variety of narrow-line region cloud origins.
\end{abstract}

Subject headings: galaxies: ISM — galaxies: Seyfert — line: formation

\section{INTRODUCTION}

The failure of "simple" photoionization models to describe the narrow emission line spectrum of active galactic nuclei (AGNs) was shown dramatically by Filippenko \& Halpern (1984), who found that the line widths of the optical forbidden lines were correlated with the critical density and ionization potential of those lines. This correlation indicates stratification of the photoionized region, with the lines emitting near their critical densities.

Recent Hubble Space Telescope (HST) images of Seyfert 2 galaxies show that these objects are rich with ionization cones (Evans et al. 1991; Tsvetanov \& Walsh 1992; Wilson et al. 1993; Macchetto et al. 1994; Arribas, Mediavilla, \& García-Lorenzo 1996), knots (Acosta-Pulidó et al. 1996), filaments, and strands (Falcke et al. 1996) of ionized gas. We should not expect that these complex environments can be described with simple, single-component photoionization models. Similar images of Seyfert 1 type objects show a less complex, symmetric structure that may indicate a viewing angle preference consistent with the unification model of Seyfert galaxies (Schmitt \& Kinney 1996).

Two-component photoionization models have been proposed by Binette, Wilson, \& Storchi-Bergmann (1996) and have met with some success in describing the emission-line

\footnotetext{
${ }^{1}$ Operated by the Association of Universities for Research in Astronomy (AURA), Inc., under cooperative agreement with the National Science Foundation.
}

spectrum of Seyfert galaxies. This model assumes that the narrow-line region (NLR) is composed of both matterbounded and ionization-bounded clouds and that the covering fraction of the two populations can account for the range of observed line emission. A multicomponent photoionization model has been proposed by Komossa \& Schulz (1997), who assume that the NLR is effectively composed of a few clouds with a range of gas densities and distances from the central AGN. Both of these types of models do much better at reproducing the scatter in the line ratio observations than single-component photoionization models.

An alternative to photoionization by the central source is photoionization by fast-moving shocks. Dopita \& Sutherland (1995) describe in detail these "photoionizing" shocks and argue that all narrow emission lines can be excited by these processes. However, Morse, Raymond, \& Wilson (1996) and Wilson (1997) discuss the viability of such shocks in describing the emission spectrum of active galaxies. In particular, Wilson (1997) argues that the energetics of radiative shocks may not account for the observed line luminosities. It is still unclear exactly what role shocks play in the NLR environment, that is, whether they provide the bulk of the energy input for the observed emission or merely compress gas so that it emits more strongly when photoionized by a central source (Pogge 1997).

We will assume that photoionization by the central source is the dominating mechanism responsible for NLR emission. We extend the locally optimally emitting clouds (LOC) hypothesis of Baldwin et al. (1995) to NLR gas. This 
assumes that, in nature, gas with a wide range of physical conditions is present and the emission-line spectrum we observe is predominately a result of a simple, yet powerful, selection effect: we observe lines from clouds best able to emit them. To this end, we present the results of a large grid of photoionization models and make predictions of the nature of the integrated spectrum.

\section{MODEL CALCULATIONS}

\subsection{Assumptions}

For simplicity, we will assume that the source of narrowline emission observed in active galaxies is from photoionized clouds that are similar to illuminated molecular clouds in our own Galaxy. The source of these clouds may be fourfold: (1) molecular cloud complexes in the disk of the galaxy, (2) the dusty molecular torus that is believed to surround the central source and that will certainly be photoionized, (3) interstellar matter (ISM) entrained behind a radio jet so that photoionization by the central source becomes important, and (4) material "snowplowed" in the form of bow shocks in front of a radio jet such that it too may be photoionized by the central source. We will ignore emission that may be due to shock excitation coming from photoionizing shocks, although how important this emission is to the overall spectrum is unclear (Wilson 1997).

We have used the spectral synthesis code CLOUDY (version 90.03; Ferland 1996) to calculate the emission from plane-parallel, constant hydrogen density clouds ionized by a continuum similar to that expected from a typical Seyfert galaxy with $L_{\text {ion }}=10^{43.5} \mathrm{ergs} \mathrm{s}^{-1}$. The shape of the ionizing continuum was chosen to be a combination of a UV bump of the form $f_{v} \propto v^{-0.3} \exp \left(-h v / k T_{\text {cut }}\right)$ and an X-ray power law of the form $f_{v} \propto v^{-1.0}$ spanning $13.6 \mathrm{eV}$ to $100 \mathrm{keV}$. The UV bump cutoff temperature, $T_{\text {cut }}$, was chosen such that the UV bump peaked (in $v F_{v}$ ) at $48 \mathrm{eV}$. The UV and X-ray components were combined with a typical Seyfert UV to $\mathrm{X}$-ray spectral slope, $\alpha_{o x}=-1.2$.

The ionization/thermal equilibrium and radiative transfer calculations for a single cloud proceeded until one of the following three conditions were met. First, the electron temperature dropped below $3000 \mathrm{~K}$. Gas with lower temperatures does not contribute significantly to the emission lines presented in this paper. Second, we do not allow clouds to have thicknesses in excess of $10 \%$ of its distance from the central continuum source. Third, we do not allow the total hydrogen column density to exceed $10^{24} \mathrm{~cm}^{-2}$; this mainly affects the very high ionization clouds and prevents them from becoming Thomson thick. In practice, the third condition had little impact on the narrow emission lines. The largest cloud of the grid presented below is one with a thickness of $\sim 16 \mathrm{pc}$, consistent with the size of large molecular clouds. We will comment further on the effects of these stopping criteria in a later section.

Finally, we assume that each cloud sees the full continuum with no obscuration. At large enough distances, intervening clouds or diffuse ISM may attenuate the ionizing spectrum significantly. We have looked at the effects of such ISM attenuation on the incident continuum: an ISM with gas densities as low as $0.1 \mathrm{~cm}^{-3}$ such as found in the local ISM of the Milky Way (Wood \& Linsky 1997) would have no effect on the results presented below. A higher density ISM $\left[n(\mathrm{H})=0.5 \mathrm{~cm}^{-3}\right]$, however, would have an effect at large distances from the ionizing source, due to the larger column density and lower ionization resulting in a higher gas opacity. For the higher density gas and the shape and luminosity of the incident continuum given above, the attenuated continuum has an optical depth of unity at 4 ryd at a distance of $R \sim 10^{21.75} \mathrm{~cm}$ from the ionizing source, resulting in 5\%-20\% changes in the line luminosities. At larger distances, the attenuation by a dense ISM would result in a decrease in the luminosity of the emitted spectrum of up to a factor of 10 . We neglect this complication for simplicity.

\subsection{Reprocessing Efficiency}

We have computed, on the University of Kentucky Convex Exemplar parallel supercomputer, a grid of 1881 of the single cloud models described above in a plane of hydrogen gas density and distance from the central ionizing source. We chose a range of parameters in order to cover all the possible physical conditions of narrow-line region gas. The hydrogen gas density, $n(\mathrm{H})$, ranged from $10^{2}$ to $10^{10}$ $\mathrm{cm}^{-3}$. The incident continuum flux was varied so that for an assumed ionizing continuum luminosity $L_{\text {ion }}=10^{43.5}$ $\operatorname{ergs~s}^{-1}$ [monochromatic luminosity $L_{\lambda}(1450 \AA)=10^{39.558}$ ergs s${ }^{-1} \AA^{-1}$ for the continuum shape used here], the distance from the ionizing source of radiation, $R$, varied from $10^{15}$ to $10^{22} \mathrm{~cm}$. This distance range scales with the luminosity as $L_{\text {ion }}^{1 / 2}$. The conversion from incident flux to luminosity and radius assumes that the ionizing radiation is emitted isotropically. However, observations of ionizing "cones" (Evans et al. 1991; Tsvetanov \& Walsh 1992; Wilson et al. 1993; Macchetto et al. 1994; Arribas et al. 1996) and UV-photon deficits (Binette, Fosbury, \& Parker 1993; Morse, Raymond, \& Wilson 1996) imply that the gas may see a different ionizing continuum than we do.

Calculations were performed assuming two abundance sets: a solar set and a set including dust that varied with distance from the ionizing source. We will describe each set of abundances in turn and compare the two in terms of diagnostic line ratio diagrams in a later section.

\subsubsection{Solar Abundances}

Figure 1 shows the results of grid calculations, for solar abundance clouds, in the form of contour plots of logarithm equivalent width referred to the incident continuum at 4860 $\AA$. The solar abundances are from Grevesse \& Anders (1989) and Grevesse \& Noels (1993) and have the following values:

$\mathrm{H}: 1.00$, He: $1.00 \times 10^{-1}$, Li: $2.04 \times 10^{-9}, \mathrm{Be}: 2.63 \times$ $10^{-11}$, B: $7.59 \times 10^{-10}, \mathrm{C}: 3.55 \times 10^{-4}, \mathrm{~N}: 9.33 \times 10^{-5}$, O: $7.41 \times 10^{-4}, \mathrm{~F}: 3.02 \times 10^{-8}$, Ne: $1.17 \times 10^{-4}, \mathrm{Na}$ : $2.06 \times 10^{-6}, \quad \mathrm{Mg}: 3.80 \times 10^{-5}, \quad \mathrm{Al}: 2.95 \times 10^{-6}, \quad \mathrm{Si}:$ $3.55 \times 10^{-5}, \quad$ P: $3.73 \times 10^{-7}, \quad$ S: $1.62 \times 10^{-5}, \quad \mathrm{Cl}$ : $1.88 \times 10^{-7}, \quad$ Ar: $3.98 \times 10^{-6}, \quad \mathrm{~K}: 1.35 \times 10^{-7}, \mathrm{Ca}$ : $2.29 \times 10^{-6}, \quad$ Sc: $1.58 \times 10^{-9}, \quad$ Ti: $1.10 \times 10^{-7}, \quad$ V: $1.05 \times 10^{-8}, \mathrm{Cr}: 4.84 \times 10^{-7}, \mathrm{Mn}: 3.42 \times 10^{-7}, \mathrm{Fe}:$ $3.24 \times 10^{-5}$, Co: $8.32 \times 10^{-8}, \mathrm{Ni}: 1.76 \times 10^{-6}, \mathrm{Cu}$ : $1.87 \times 10^{-8}, \mathrm{Zn}: 4.52 \times 10^{-8}$.

Furthermore, we assume that there is no dust present in the solar abundance computations.

The ionization parameter provides a useful homology relationship with the emission lines (Davidson 1977). $U(\mathrm{H})$ is defined as the ratio of hydrogen ionizing photon density to hydrogen density, $U(\mathrm{H}) \equiv \Phi(\mathrm{H}) / n(\mathrm{H}) c$, where $\Phi(\mathrm{H})$ is the flux of hydrogen ionizing photons and $c$ is the speed of light. For reference, a dashed line is placed in the upper 


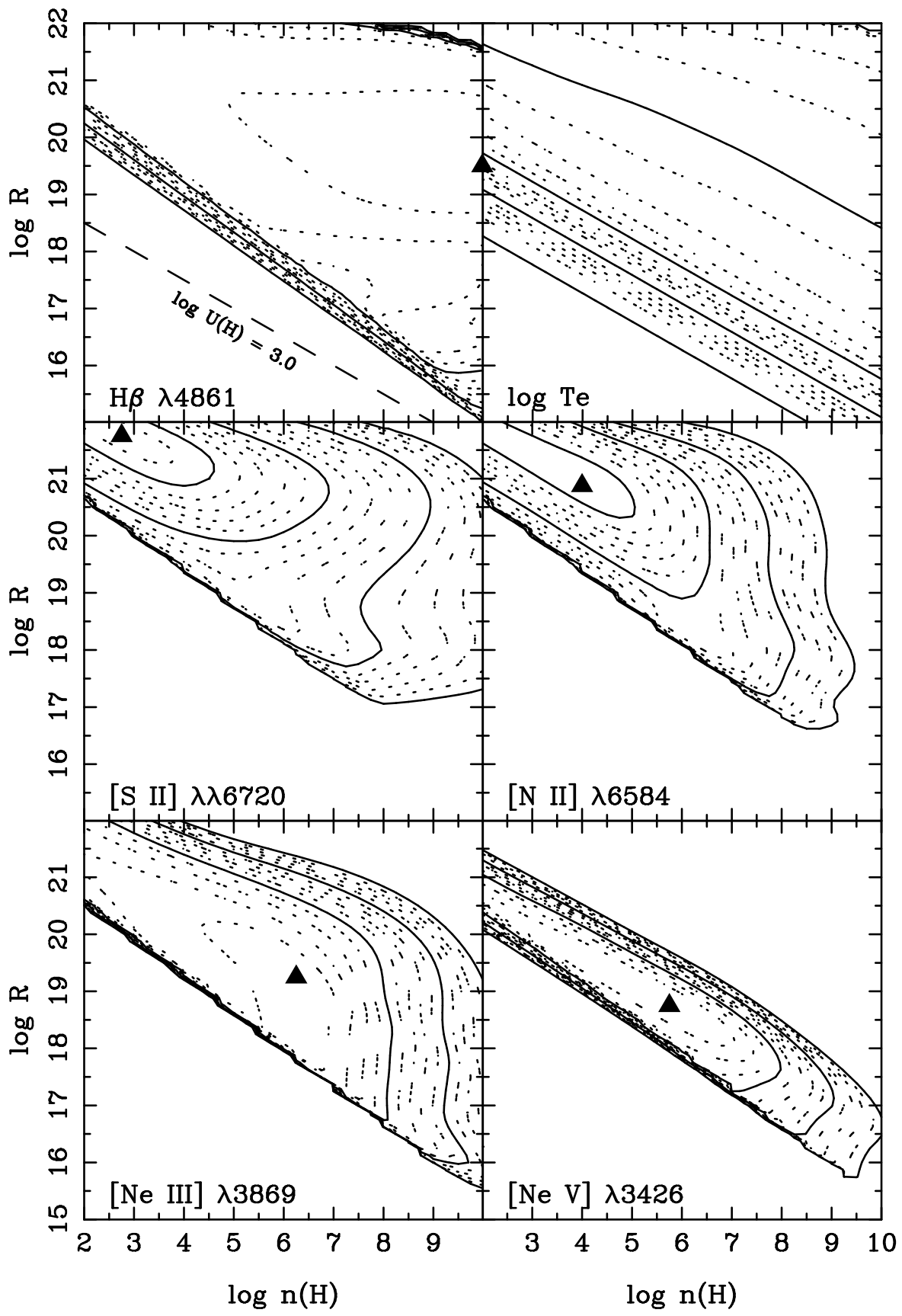

FIG. $1 a$

FIG. 1. - Contours of constant logarithmic line equivalent widths as a function of $\log R$ and $\log n(\mathrm{H})$ for the 23 ions indicated, referenced to the incident continuum at $4860 \AA$. The bold lines represent 1 dex increments, and the dotted lines are 0.2 dex steps. The triangle is the peak of the equivalent width distribution, and the contours decrease downward to the outer value of $1 \AA$. The reader will sometimes find it convenient to view the contour plots along the ridge at large inclination angle to the sheet of paper. The upper right-hand plot in panel $a$ is the $\log T_{e}$ of the front face of the cloud. The temperature decreases from $10^{7} \mathrm{~K}$ in the lower left-hand corner of the plot to $10^{3} \mathrm{~K}$ in the upper right-hand corner. Again, the bold lines represent 1 dex increments, and the dotted lines are 0.2 dex steps.

left-hand plot of Figure $1 a$ representing $\log U(\mathrm{H})=3.0$. The ionization parameter increases from top right to bottom left in these diagrams; lines of constant $U(\mathrm{H})$ have a slope of $d \log R / d \log n=-0.5$.

Shown in Figure 1 are 23 strong optical, UV, and infrared recombination, resonance, and forbidden lines. The temperature of the illuminated face of the cloud is also shown in the upper right-hand plot of Figure 1a. For many emission lines, a ridge of near maximum equivalent width runs diagonally across the distance-gas density plane, roughly parallel to lines of constant ionization parameter. Most lines are emitted optimally for a narrow range of ionization parameter spanning this ridge. For larger (smaller) ionization parameters, the gas is overionized (underionized), and the line is not efficiently emitted. This explains the sudden drops in the emission-line equivalent widths on either side of their ridges. Moving along the ridge to increasing gas densities, at near constant ionization parameter, the forbidden lines become collisionally deexcited and the line equivalent width falls off. Moving along the ridge at con- 


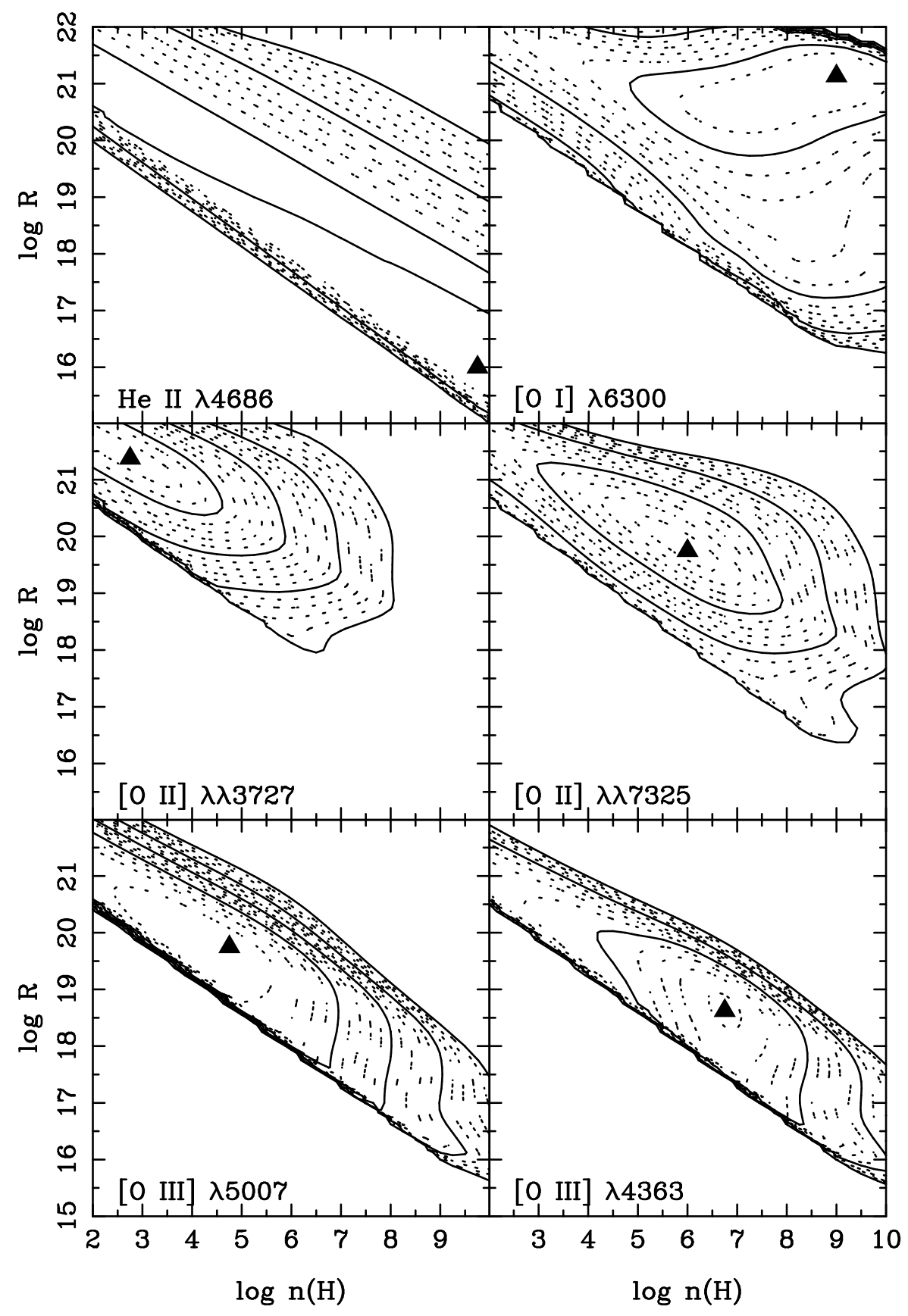

FIG. $1 b$

Fig. 1-Continued

stant ionization parameter to smaller gas densities, the equivalent widths of some lines also diminish as other lines of similar ionization, but lower critical density, become important coolants. This effect is especially dramatic for $\mathrm{C}$ IV $\lambda 1549$ and $\mathrm{O}$ VI $\lambda 1035$.

The stopping criteria assumed in $\S 2.1$ do affect the resulting spectrum. The grid of models shown in Figure 1 includes clouds that are both optically thin and optically thick to hydrogen ionizing radiation. Optically thin clouds generally occupy the lower left-hand portion of the distance-density plane below a line with slope $d \log R / d \log$ $n \sim-\frac{2}{3}$ and intersecting the lower right-hand corner. The temperature of this gas (see Fig. 1) ranges from $20,000 \mathrm{~K}$ to several million K. Emission lines presented in this paper are not emitted by gas with $T_{e} \gtrsim 10^{5} \mathrm{~K}$ (X-ray lines are). These hot clouds are generally truncated by the plane-parallel condition discussed earlier. Optically thick clouds are in the upper right-hand portion of the figures, and the gas generally has nebular temperatures, except the extreme upper right-hand corner, where the electron temperatures can be as low as a few hundred $\mathrm{K}$ and the gas is mostly molecular. Generally, the optically thick clouds were stopped because the electron temperature fell below $3000 \mathrm{~K}(\S 2.1)$. The total hydrogen column density restriction mentioned in $\S 2.1$ applies to a small number of high-density optically thick clouds in a triangular region bounded by a distance of $1 \mathrm{pc}$ 


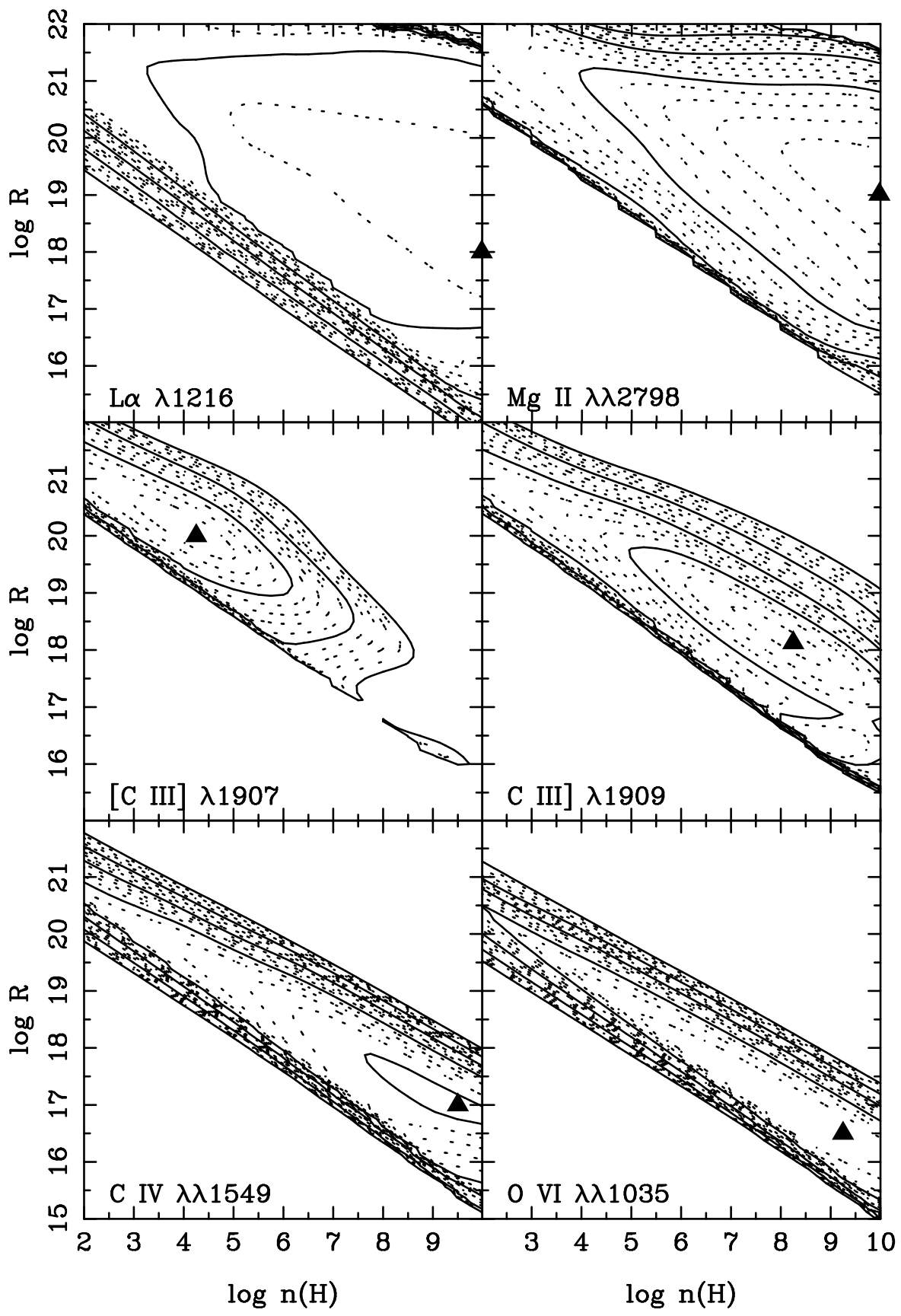

Fig. $1 c$

FIG. 1-Continued

on the top and a line roughly parallel to $\log U \sim-1.0$ on the left-hand side. The clouds in this region do not significantly contribute to the narrow forbidden line spectrum.

Different types of lines can be seen in Figure 1 to be emitted from clouds with a range in gas densities and distances. Recombination lines, such as $\mathrm{H} \beta$ and $\mathrm{Ly} \alpha$, generally form broad planes of maximum equivalent width, whereas the forbidden lines, such as $[\mathrm{Ne} \mathrm{v}]$, are either narrow ridges or peaked islands ([O II $]$ 27325). Figure $1 b$ shows the differences in the nebular and auroral lines of [O II] and [O III]. Notice that the auroral features are formed optimally at higher densities, because of their higher critical density. Figure $1 c$ shows the contour plots of a few of the stronger UV lines. Figure $1 d$ shows a few strong $[\mathrm{Fe}$ II] infrared fine-structure lines from a 16 level model atom. The model atom has the same configuration as that shown in Thompson (1995) and uses the collision data of Pradhan \& Zhang (1993) and Einstein $A$-coefficients from Quinet, Le Dourneuf, \& Zeippen (1996). Verner et al. (1997) compare the differences between the 16 level model atom and a much larger definitive 371 level calculation. Preliminary comparisons show that predicted line intensities of the 16 level atom differ typically by a few percent and at maximum by $10 \%$ from the larger 371 level calculation.

To illustrate the problems encountered when trying to use conventional diagnostic line intensity ratios for an ensemble of clouds with a wide range of physical conditions, we momentarily assume that each emission line in the total 


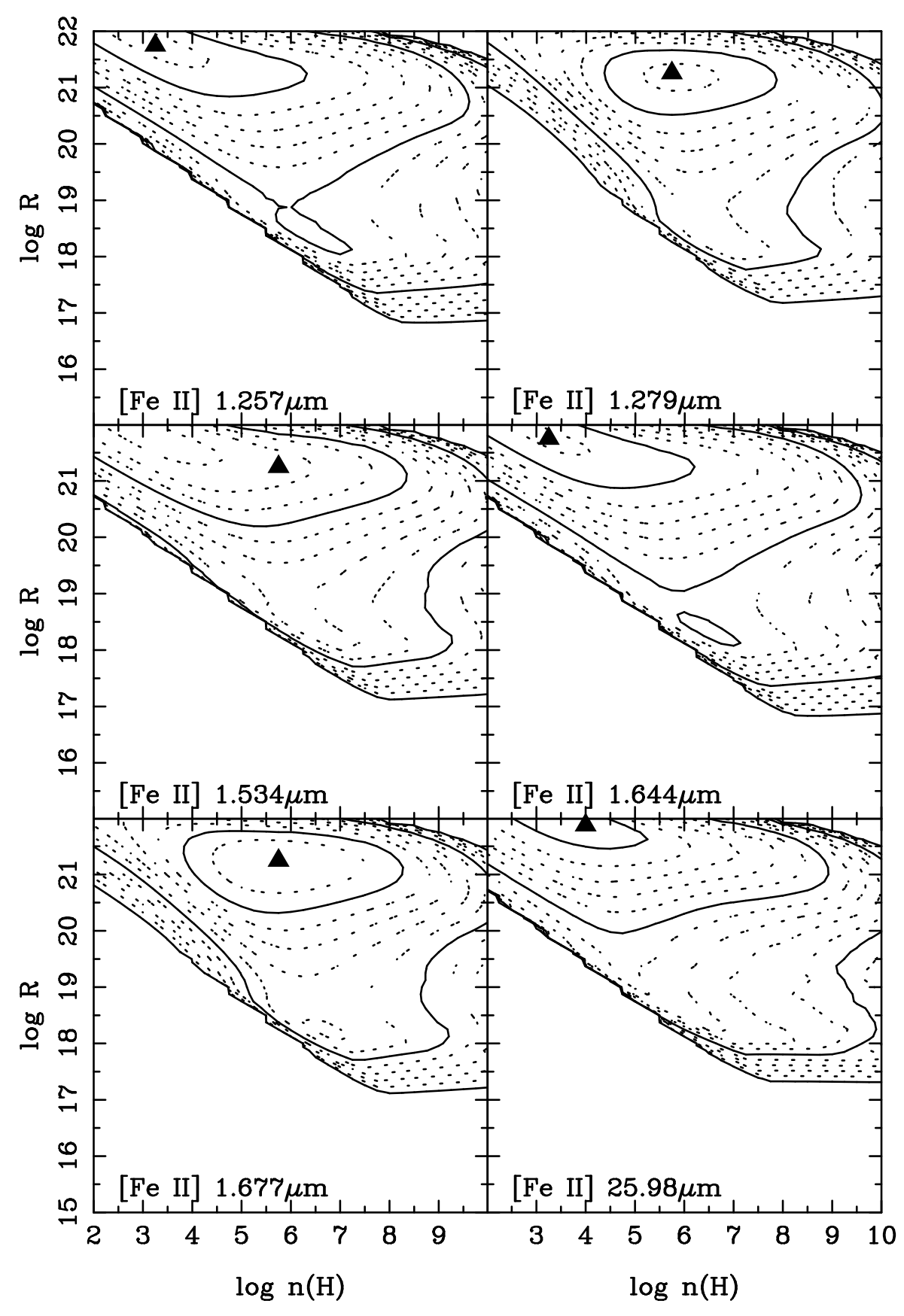

FIG. $1 d$

FIG. 1-Continued

spectrum is produced with the maximum possible equivalent width found in the figures. We then use this spectrum to reproduce the standard density and temperature diagnostic line ratios. This is the simplest way to document the effects of a distribution of clouds. Three density indicators are given by Osterbrock (1989). These include the [S II] $26716 /$ $\lambda 6731$ and [O II] $\lambda 3729 / \lambda 3726$ doublets and the [C III] $\lambda 1907 / \mathrm{C} \mathrm{III}] \lambda 1909$ ratio. For [Fe II], Pradhan \& Zhang (1993) use the $1.534 / 1.644 \mu \mathrm{m}$ ratio as a density indicator. Assuming a temperature of $10,000 \mathrm{~K}$, the [O II] ratio we predict gives an $n_{e}$ of $300 \mathrm{~cm}^{-3}$, for the predicted [S II] doublet the density is $700 \mathrm{~cm}^{-3}$, for the $\left[\mathrm{Fe} \mathrm{III}\right.$ ratio $n_{e}$ is $20,000 \mathrm{~cm}^{-3}$, and the $[\mathrm{C} \mathrm{III]} / \mathrm{C} \mathrm{III]}$ ratio gives a density of nearly $300,000 \mathrm{~cm}^{-3}$. The $[\mathrm{O}$ III] $(\lambda 4959+\lambda 5007) / \lambda 4363$ temperature-density diagnostic using the peak emission of Figure 1 and an electron temperature of $10,000 \mathrm{~K}$ gives an electron density on the order of a few million $\mathrm{cm}^{-3}$. The point of this exercise is clear: line diagnostics fail when the gas has a wide range of physical conditions. As Figure 1 shows, many of these lines form at different densities and temperatures. However, by using the diagnostic line ratios, one assumes that the lines are formed in gas of similar physical conditions, which is obviously not the case in Figure 1 and is likely not the case in nature. In a later section, we will integrate the "visibility functions" of Figure 1 to calculate an emission spectrum by assuming that the observed spectrum is the result of an ensemble of clouds with differing properties. 


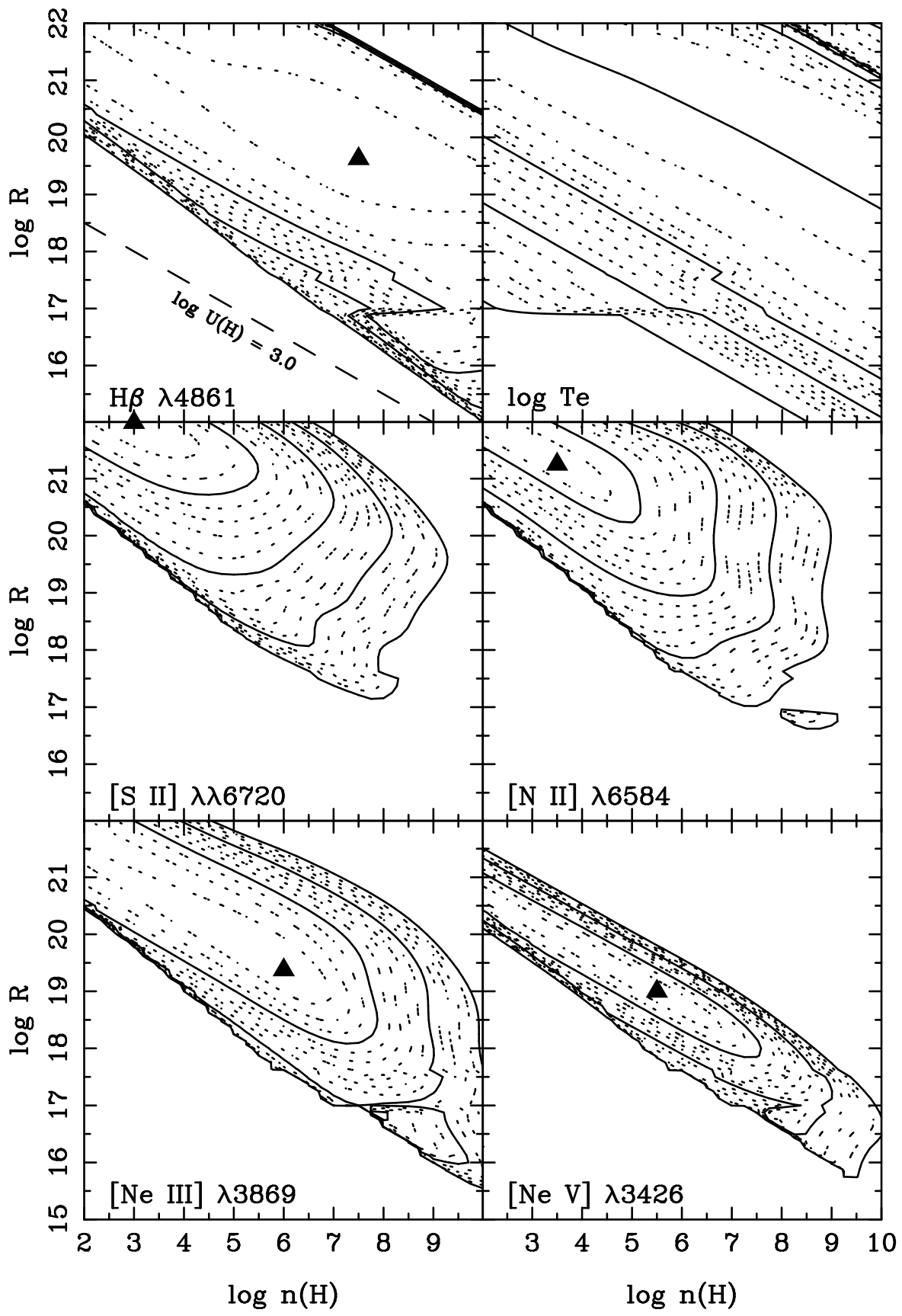

FIG. $2 a$

FIG. 2.-Same as Fig. 1, except for the dusty abundance set described $\S 2.2 .2$

\subsubsection{A Dusty NLR}

In the previous section we have assumed that the narrowline region is a dust-free environment. We now recompute the grid assuming that the clouds are dusty. Grains will not survive in an environment with high photoionizing flux because of the various sublimation processes discussed by Laor \& Draine (1993). Therefore, after the manner of Netzer $\&$ Laor (1993), we vary the abundances as a function of distance from the ionizing source, in order to mimic these destruction processes. At small distances from the central source, we assume the abundances are solar, as given in the previous section. At a distance of approximately $10^{16.9} \mathrm{~cm}$ from the central source (for the ionizing luminosity given above), Orion-type graphite grains (Baldwin et al. 1991) are just at their sublimation temperature $(\sim 1750 \mathrm{~K})$ at the cloud face. At distances larger than this, we include graphite grains within the calculation of the single cloud and deplete carbon such that its abundance is typical of an $\mathrm{H}$ II region (see below and Baldwin et al. 1996). Orion-type silicate grains sublimate at $\sim 1400 \mathrm{~K}$, corresponding to a distance of $10^{17.6} \mathrm{~cm}$. Beyond this distance, the abundances are set to values that approximate those found in the Orion nebula:

$\mathrm{H}: \quad 1.00, \mathrm{He}: 9.50 \times 10^{-2}, \quad \mathrm{C}: 3.00 \times 10^{-4}, \quad \mathrm{~N}$ : $7.00 \times 10^{-5}, \quad$ O: $4.00 \times 10^{-4}, \mathrm{Ne}: 6.00 \times 10^{-5}, \mathrm{Na}:$ $3.00 \times 10^{-7}, \quad \mathrm{Mg}: 3.00 \times 10^{-6}, \quad \mathrm{Al}: 2.00 \times 10^{-7}, \quad \mathrm{Si}:$ $4.00 \times 10^{-6}, \quad$ S: $1.00 \times 10^{-5}, \quad \mathrm{Cl}: 1.00 \times 10^{-7}, \quad \mathrm{Ar}:$ $3.00 \times 10^{-6}, \quad \mathrm{Ca}: 2.00 \times 10^{-8}, \mathrm{Fe}: 3.00 \times 10^{-6}, \quad \mathrm{Ni}$ : $1.00 \times 10^{-7}$. 


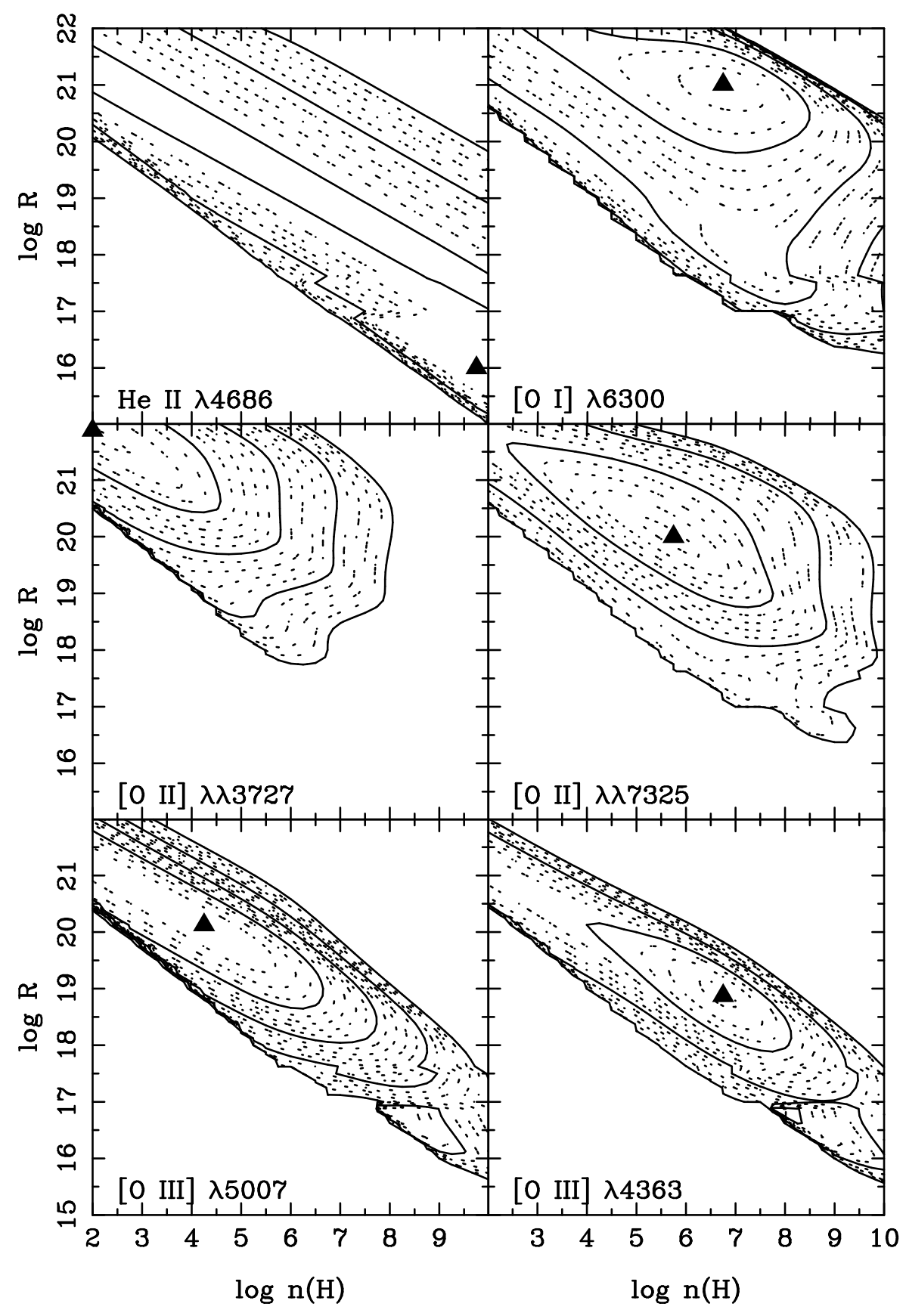

FIG. $2 b$

Fig. 2-Continued

These abundances are based on the results of several recent studies of the Orion nebula (Baldwin et al. 1991; Rubin et al. 1991, 1992; Osterbrock, Tran, \& Veilleux 1992). Although this discontinuous "turning on" of grains is unphysical and our choices of grain composition and gas depletions are specific, it serves as an example to simulate the presence of dust in the narrow-line region. The grain physics used here is described by Baldwin et al. (1991).

Figure 2 shows the same contour plots as Figure 1, except that the individual models include the grains as specified above. The discontinuous grain sublimation distances are easily seen in the figure as sharp discontinuities in the contour plots at $R=10^{16.9}$ and $10^{17.6} \mathrm{~cm}$.
Comparisons of Figures 1 and 2 show four major effects of grains: (1) the depletion of refractory elements; (2) the weakening of emission lines due to absorption of the incident continuum by dust at large $U(\mathrm{H})$; (3) the photoelectric heating of the gas by grains; and (4) in the case of resonance lines such as $\operatorname{Ly} \alpha \lambda 1216$ and $C$ IV $\lambda 1549$, line destruction by grains.

Higher ionization lines, such as [O III], [Ne III], and $[\mathrm{Ne}$ v], tend to be emitted less efficiently than in the dustfree models, with the equivalent width falling by as much as a factor of 2 . The weakening of these lines is caused by the absorption of the incident continuum by the dust. The lower ionization lines ([S II], [O II $],[\mathrm{N} \mathrm{III}],\left[\begin{array}{ll}\mathrm{I}\end{array}\right]$, etc.) 


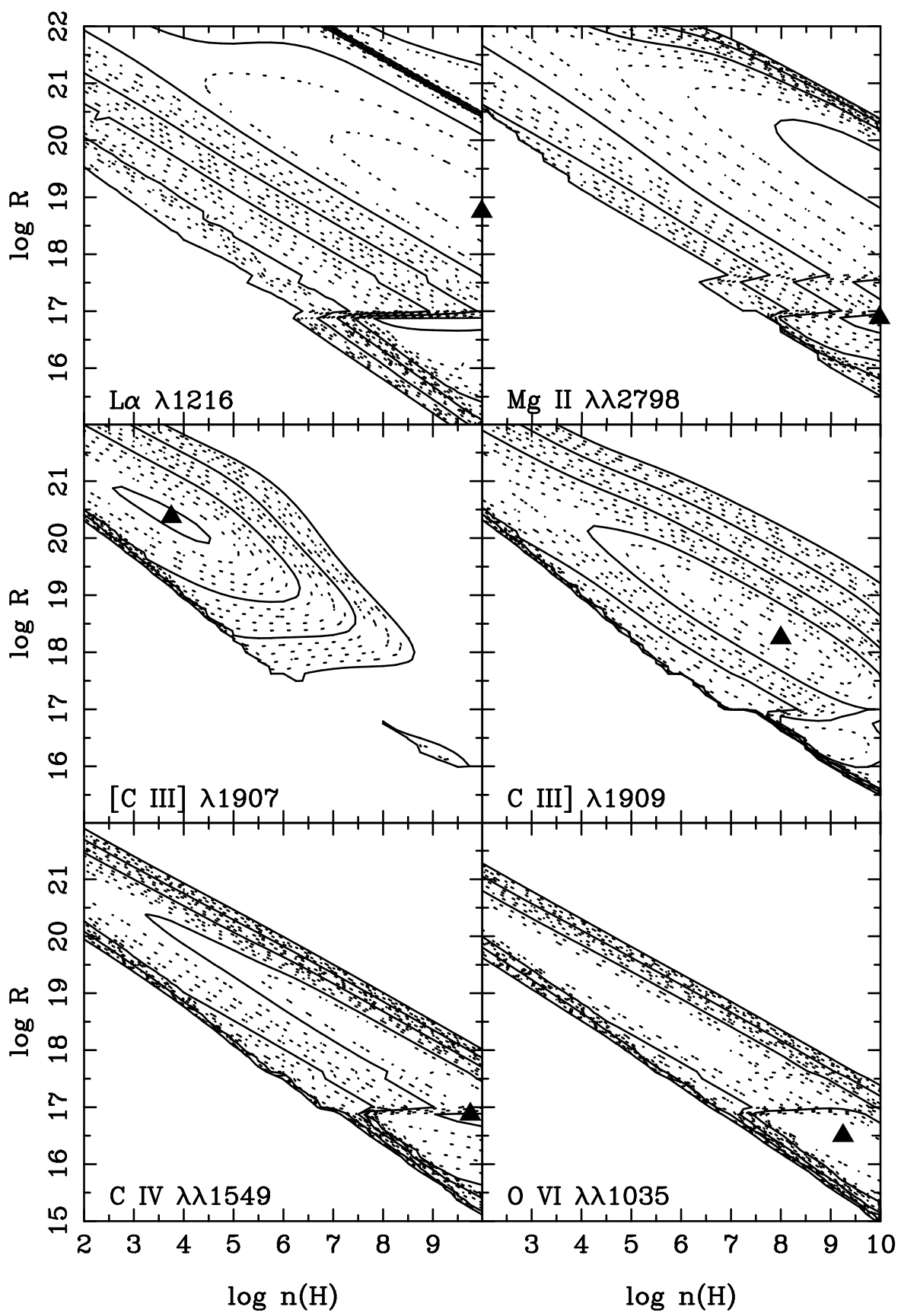

FIG. $2 c$

FIG. 2-Continued

actually have their peak emission increase, though at lower ionization parameters, because of the photoelectric heating of the gas by the grains. Resonance line destruction is readily apparent in Figure $2 c$. The heavy depletion of iron is also apparent in Figure $2 d$, wherein the peak equivalent width drops by nearly a factor of 10 . This last point will be discussed more fully in a later section.

\section{INTEGRATED LINE EMISSION}

The equivalent widths shown in Figures 1 and 2 reflect the efficiency with which the clouds reprocess the incident continuum. It is clear that different lines are formed optimally in different places in the gas density-distance plane. If the distribution function of clouds in this plane is known, then the total line luminosity emitted by a set of clouds will be given by

$$
L_{\text {line }} \propto \iint r^{2} F(r, n) \psi(r, n) d n d r,
$$

where $F(r, n)$ is the emission line flux of a single cloud at radius $r$ and gas density $n$ and $\psi(r, n)$ is the cloud distribution function, which is not necessarily analytical.

The remaining question is the spatial distribution of the NLR clouds. Many type 2 Seyfert galaxies have resolved NLRs, often with complex structures that are associated with linear or double-lobed radio sources (Bower et al. 


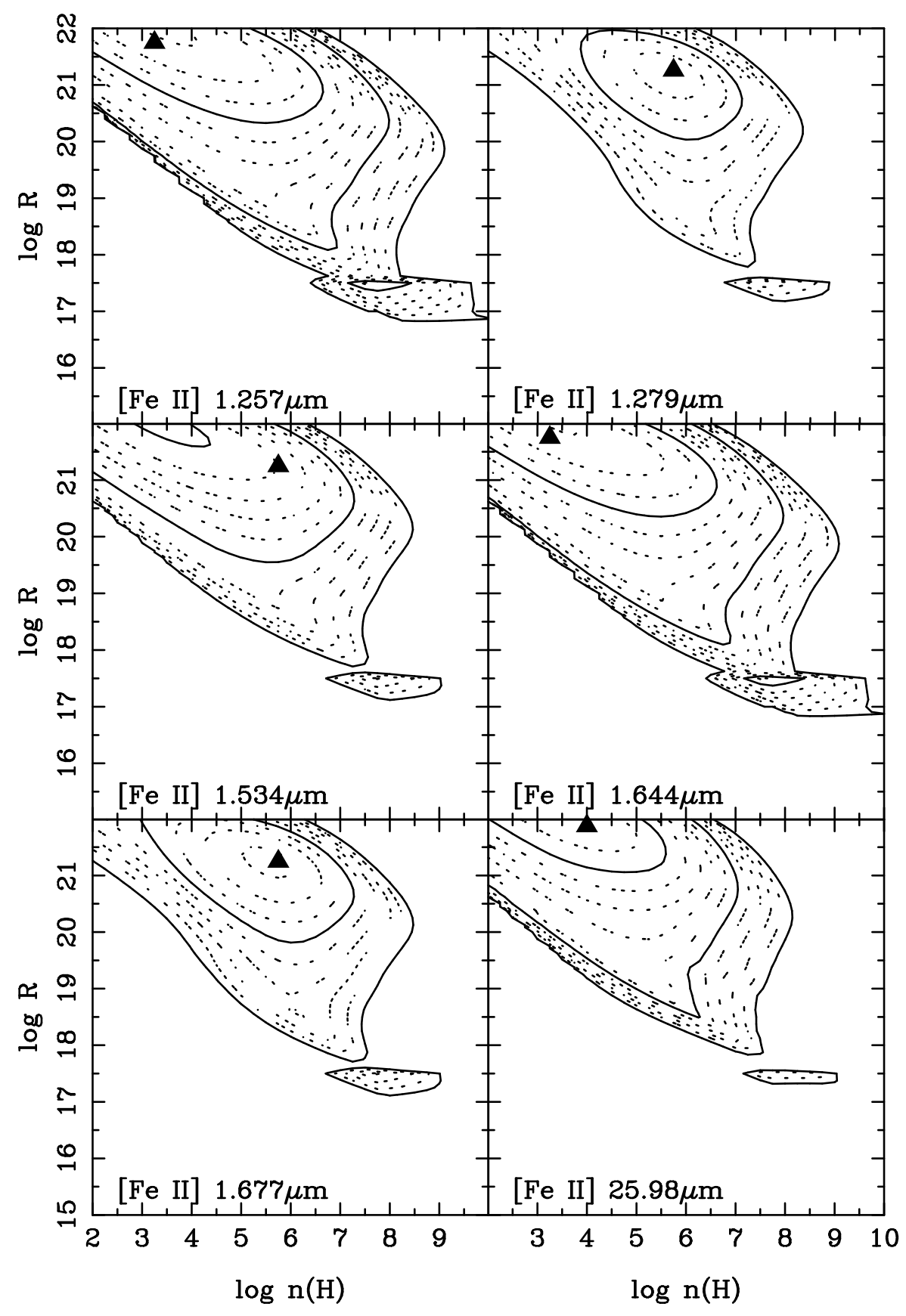

FIG. $2 d$

FIG. 2-Continued

1995; Pogge \& De Robertis 1995; Capetti et al. 1996; Cooke et al. 1997), whereas the structure of the NLR in type 1 Seyfert galaxies is generally more compact and axisymmetric, at least in projection (Schmitt \& Kinney 1996; Nelson et al. 1996). Since it is not clear how the emitting gas is distributed, in all its dimensions, we will assume that the distribution function $\psi(r, n)$ can be approximated by $\psi(r, n) \propto f(r) g(n)$, where $f(r)$ and $g(n)$ are the cloud covering fractions with distance and gas density, respectively (see Baldwin et al. 1995). For simplicity, we assume simple power laws:

$$
f(r) \propto r^{\gamma}, \quad g(n) \propto n^{\beta} .
$$

The power-law indices, $\gamma$ and $\beta$, will be the only free parameters of our predicted integrated spectra. In the case where both weighting functions are proportional to the inverse of $n$ or $r$, then the line equivalent widths of the clouds are equally weighted (in log space) across the gas densitydistance plane.

In the integrations computed below, we assumed certain integration limits in the gas density-distance plane in order to exclude gas that may not be present in the NLR. We exclude gas with densities $\log n(\mathrm{H})>8$, the rough maximum critical density of the optical forbidden lines observed. By including gas with $\log n(\mathrm{H}) \sim 9$, the line ratios do not change significantly except for those integrations 
that are weighted to higher densities [flatter $g(n)$ ]. If present, the contribution of high-density gas in the inner regions of the NLR cannot be significant or else the narrow-line spectrum would appear vastly different (forbidden lines would be too weak relative to the hydrogen lines). We exclude gas that is closer than $0.1 \mathrm{pc}$ to the ionizing source. This distance corresponds to the grain sublimation point described in $\S 2.2 .1$. Integration limits were also included in ionization parameter space in order to exclude gas that is far overionized (underionized) and does not contribute to the observed emission. These limits effectively exclude gas from the broad emission line region and very high density gas hundreds of parsecs away from the central engine.

\subsection{Diagnostic Line Ratio Diagrams}

Integrations described above have been computed and plotted after the manner of Baldwin, Phillips, \& Terlevich (1981, hereafter BPT). Intensity ratios for the integrated model spectra are plotted in this manner in Figure 3. Two types of lines are shown in the figure. Solid lines represent dusty models with depleted refractory elements typical of $\mathrm{H}$ II region type abundances (hereafter "dusty"). The dashed lines represent dust-free clouds with solar metallicities (hereafter "dust free"). Different values of $g(n)$ (cf. eq. [2]) are represented by different lines, with the power of $f(r)$ lying in the range $-2.0 \leq \gamma \leq 1.0$ in 0.25 increments along each line with the direction of more negative $f(r)$ shown by the arrows. For the dusty simulations (solid lines), three values of the $g(n)$ index are shown $(\beta=-1.0,-1.6,-1.8)$, and the trend of more negative $\beta$ are indicated by the appropriate arrows in the figures. For the dust-free simulations (dashed lines), the $g(n)$ index are $\beta=-1.0,-1.4$, -1.8 ; similarly, the trends for the more negative index are indicated. The " $S$ " and " $L$ " indicate in the figures the average values of the line ratios for Seyfert, taken from Ferland \& Osterbrock (1986), and LINER-type spectra, taken from Netzer (1990), with the exceptions of He II $\lambda 4686$, [O III] $\lambda 4363$, from Ho, Filippenko, \& Sargent (1996) for the LINER galaxy M81. The observed range in [O II] $\lambda 7325$ and $[\mathrm{S} \mathrm{III]} \lambda \lambda 9069,9532$ strengths were taken from Dopita \& Sutherland (1995).

To make it possible to follow particular models from panel to panel, we have marked two fiducial models in Figure 3 . The stars represent a dusty model (solid lines) with

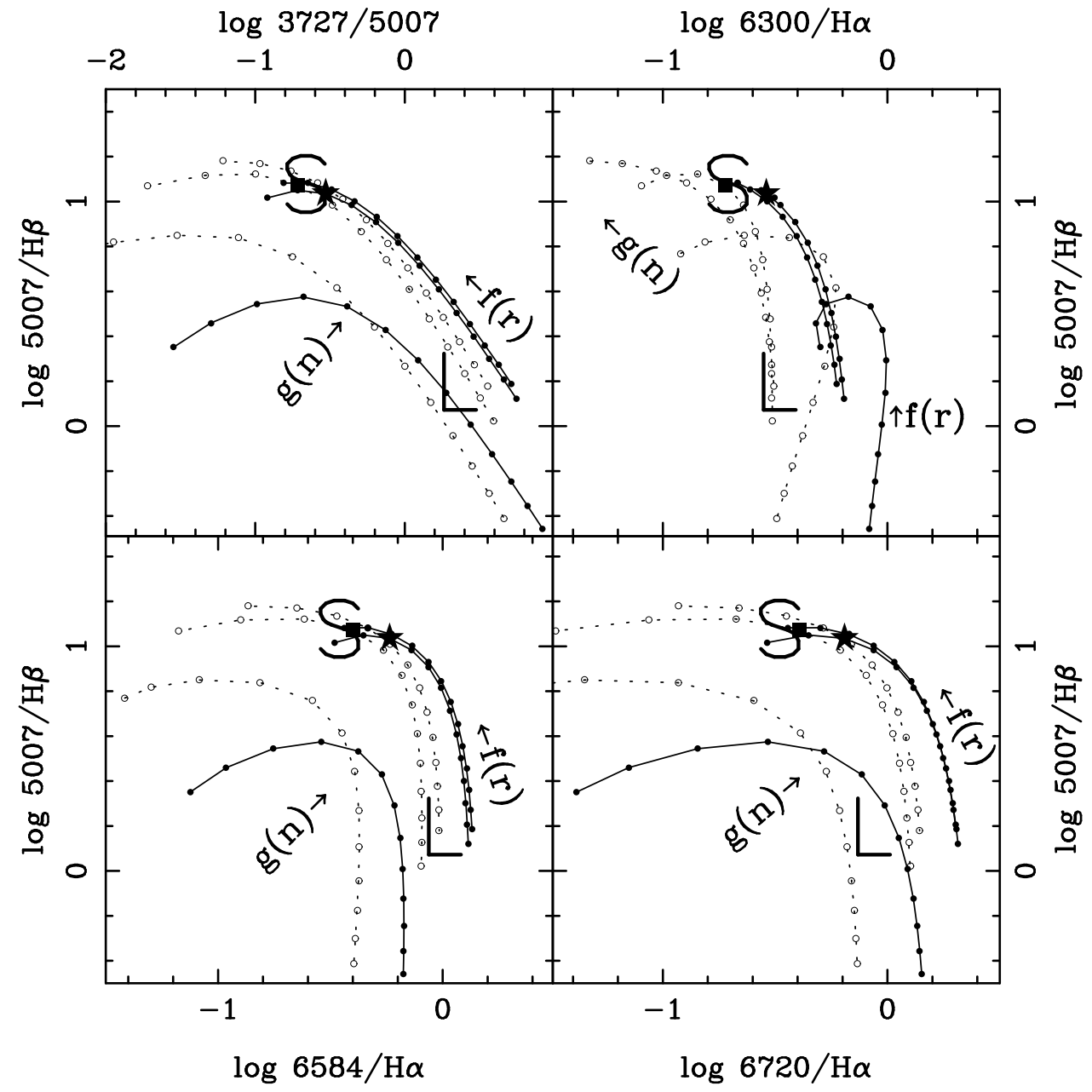

FIG. $3 a$

Fig. 3.-Line diagnostic diagrams after the manner of Baldwin, Phillips, \& Terlevich (1981). Different line types represent different abundance types: dashed lines are solar or dust free, and solid lines are the dusty abundance set described in the text. Different lines represent different families of the density weighting index, $\beta$, with different covering fraction indices, $\gamma$, along each line, with the arrows indicating the direction of the more negative index. The star represents the same model integration of dusty abundances with indices $f(r) \propto r^{-1.5}$ and $g(n) \propto n^{-1.6}$. The square represents the same model integration with solar abundances with indices $f(r) \propto r^{-1.25}$ and $g(n) \propto n^{-1.4}$. The large "S" and " $\mathrm{L}$ " symbols refer to the observations of the mean Seyfert type 2 objects and LINER type objects, respectively, as discussed in $\S 3.1$. 


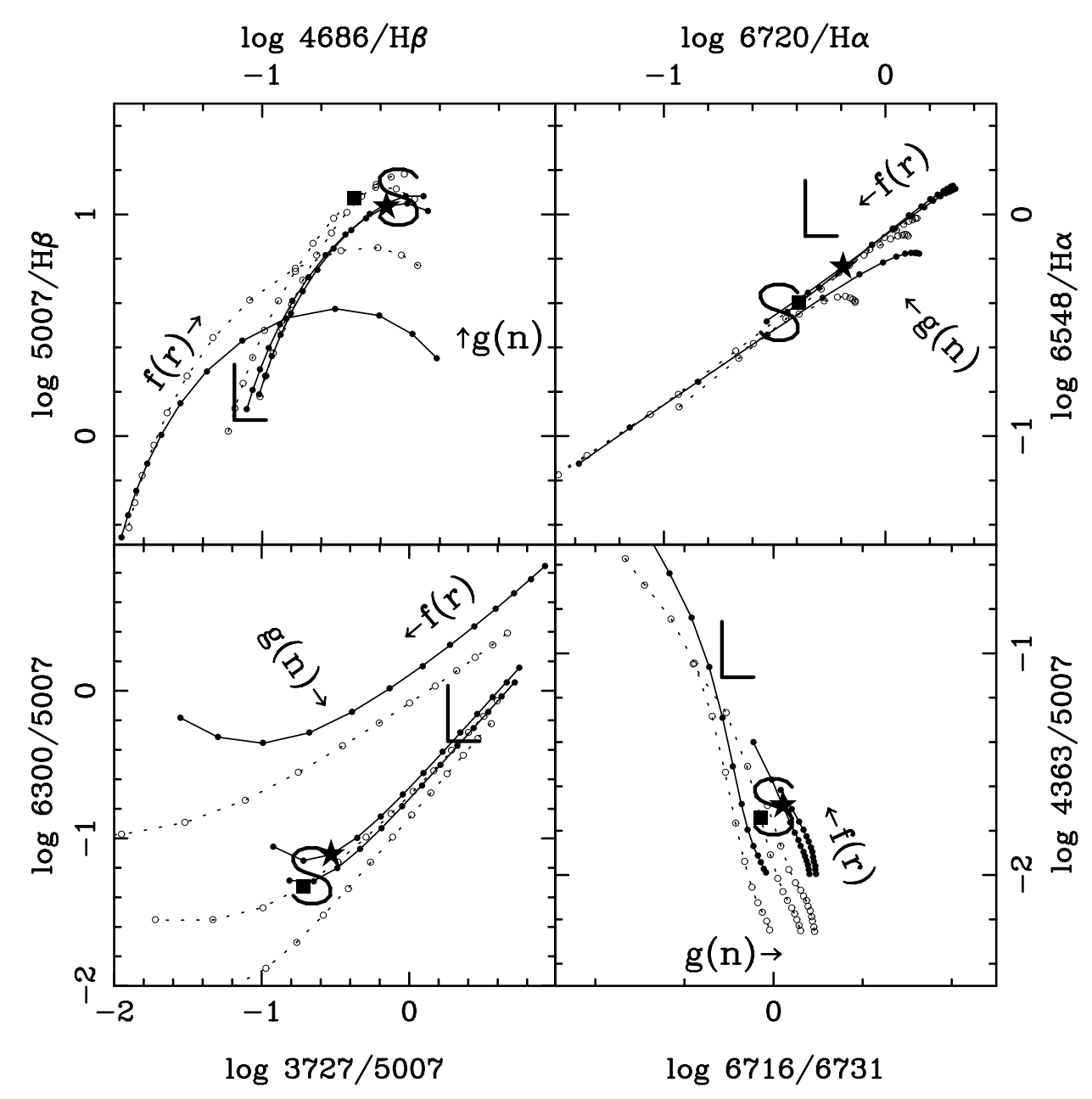

FIG. $3 b$

FIG. 3-Continued

$f(r) \propto r^{-1.5}$ and $g(n) \propto n^{-1.6}$, while the squares represent a dust-free model (dashed lines) with $f(r) \propto r^{-1.25}$ and $g(n) \propto n^{-1.4}$. These particular models were chosen because they approximately reproduce the [O $\mathrm{II}] \lambda 3727 /[\mathrm{O}$ III] $\lambda 5007$ and $[\mathrm{O} \mathrm{III}] \lambda 5007 / \mathrm{H} \beta$ line ratios in the average Seyfert 2 spectrum.

Interestingly, solar abundance, grainless calculations are not excluded by the line ratios presented in Figure 3. The dashed, dust-free, lines in Figure 3 tend to converge in the area of Seyfert observations. The dusty integrations tend to be shifted toward and into the LINER region of the diagrams, that is, with higher [N II], [S II], and [O I] relative to $\mathrm{H} \alpha$ and higher [O II] relative to [O III].

Table 1 compares the fiducial models represented by the star and square in Figure 3 with the observed average Seyfert 2 spectra from Ferland \& Osterbrock (1986), Dopita \& Sutherland (1995), Thompson (1995), and Villar-Martín \& Binette (1997). The UV/optical line ratios given by Ferland \& Osterbrock likely suffer from inaccurate absolute optical spectral flux calibrations coupled with substantially different aperture sizes between the IUE UV spectra and ground-based optical spectra coupled with the extendedness of the NLR, making many of these UV/optical narrow-line ratios highly uncertain. The table also lists the observed range of equivalent widths of $\mathrm{H} \beta$ taken from Netzer (1990) and Binette, Fosbury, \& Parker (1993).
In column (4) of Table 1 we give the dereddened UV/ optical HST Faint Object Spectrograph spectrum of the Seyfert 2 galaxy NGC 3393, from Cooke et al. (1997). The Cooke et al. observations were taken at two overlapping but significantly different aperture positions within a complicated two-dimensional line-emitting structure; spectra covering $1100-2300 \AA$ were taken at one position, while the range $2300-6800 \AA$ was covered at the other position. Because of the unintended positional offset, it is impossible to unambiguously join together the two spectral regions. We have normalized the two sections of the NGC 3393 spectrum so that the UV/optical He II ratios would agree with case B predictions. This does not guarantee consistency among the other emission lines, but it is not clear that the uncertainty due in this case to a 0 ". 4 offset between two $1^{\prime \prime}$ diameter apertures is any worse than the errors incurred through the comparison of $I U E$ to ground-based data, whereas the signal to noise ratio in the NGC 3393 spectra is much higher than in the earlier data.

Observations by Oliva et al. (1994) of the Seyfert type 2 Circinus galaxy are given in column (5) of Table 1. These observations include the ground-based optical and infrared spectra of many high-ionization "coronal lines" (ionization potentials greater than $100 \mathrm{eV}$ ).

Columns (6) and (7) of Table 1 show the results of the predicted integrated line spectrum for the dust-free and the 


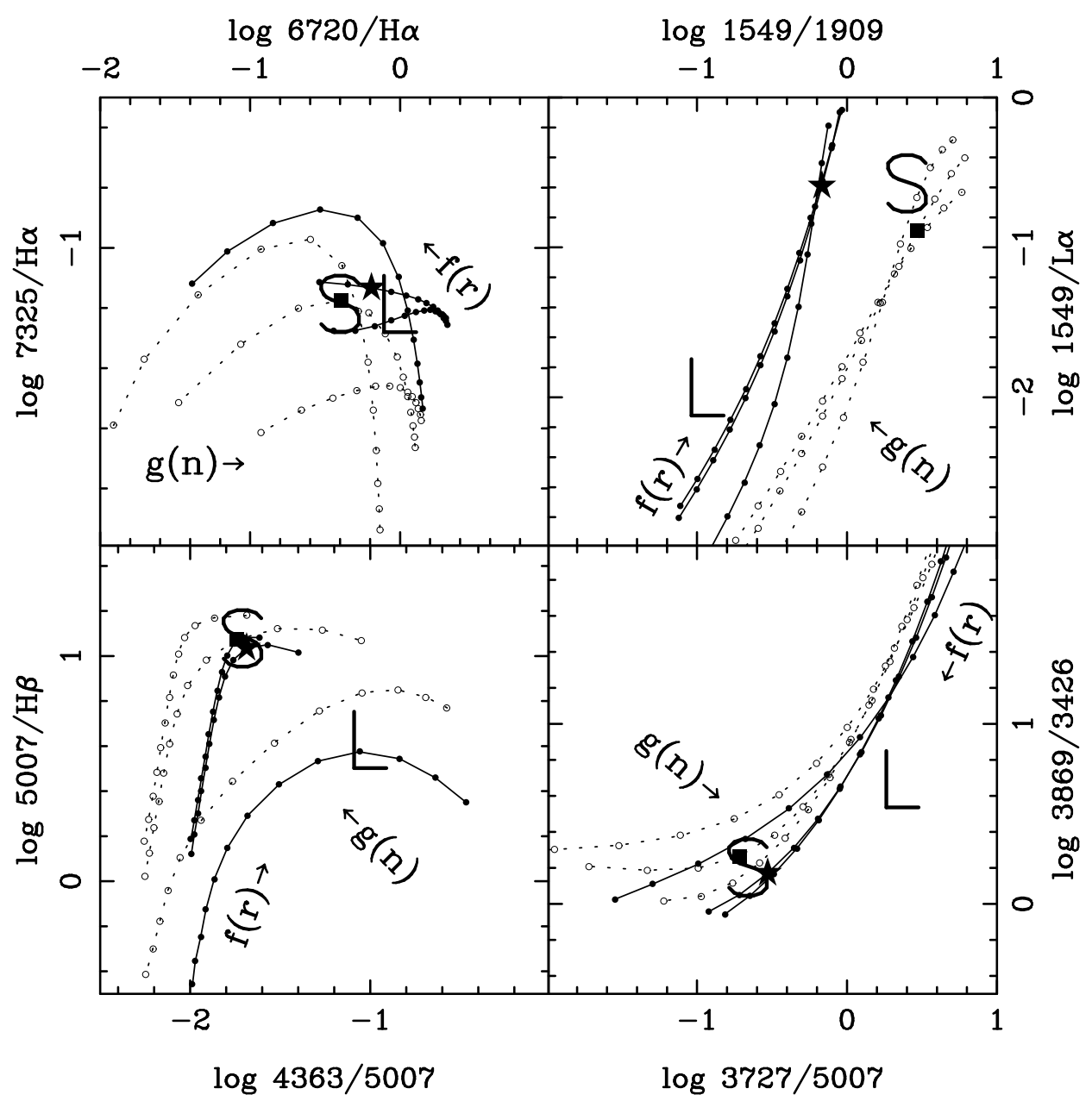

FIG. $3 c$

Fig. 3-Continued

dusty fiducial points. Recall that the integration parameters for the two fiducial models were chosen to simultaneously reproduce the $[\mathrm{O} \mathrm{III}] /[\mathrm{O} \mathrm{II}]$ and $[\mathrm{O} \mathrm{III}] / \mathrm{H} \beta$ emission line ratios of the average Seyfert galaxy, and with this in mind, the integrated spectrum is representative of the observed values.

\subsubsection{UV Lines}

Compared with the mean Seyfert 2 spectrum, the UV lines, $\mathrm{L} \alpha, \mathrm{C}$ IV, and $\mathrm{C}$ III], are generally underpredicted by the solar, dust-free integration shown in column (5) of Table 1. In column (6) of the table, the dusty integration underpredicts $\mathrm{Ly} \alpha$, and $\mathrm{C}$ IV even worse than the dust-free case (generally because of resonance line destruction by dust grains). The exceptions being that $\mathrm{C}$ III] $\lambda 1909$ line is within the observed range and $\mathrm{Mg}$ II is on the low end of the range. The predictions of the weaker UV emission lines observed in NGC 3393, not included in the mean Seyfert 2 spectrum of Table 1, are generally consistent with the observations. The exceptions $\mathrm{N} \mathrm{v}$ and $\mathrm{He}$ II will be elaborated on in the next section.

The integrations of the dust-free clouds predict $\mathrm{Ly} \alpha /$ $\mathrm{H} \beta \sim 28$, which is close to the high-density case B limit $(\sim 34)$, while the observations compiled by Ferland \& Osterbrock (1986) suggest much larger ratios (30-70). Our calculations find a maximum $\mathrm{Ly} \alpha / \mathrm{H} \beta \sim 100$ occurring at $\log n(\mathrm{H})=9.75 \mathrm{~cm}^{-3}$. Ly $\alpha / \mathrm{H} \beta$ significantly larger than $\sim 34$ implies the existence of a substantial population of high-density clouds; more specifically, $40 \leq \mathrm{Ly} \alpha / \mathrm{H} \beta \leq 70$ occurs for $5.5 \leq \log n(\mathrm{H}) \leq 8.5$. However, the spectrum resulting from integrations that heavily weight this dense gas would not be consistent with the strong optical forbidden line strengths relative to $\mathrm{H} \beta$.

Our predicted hydrogen line spectrum is in better agreement with the HST data of Cooke et al. (1997; see our Table 1). The corrected observed $\mathrm{Ly} \alpha / \mathrm{H} \beta \sim 19$ is inconsistent with the compilations of Ferland \& Osterbrock (1986), with the caveat that this observed ratio is somewhat uncertain. $\mathrm{Ly} \alpha / \mathrm{H} \beta$ ratios smaller than 23 are not possible, unless Ly $\alpha$ is weak because of either very dense $\left(>10^{11} \mathrm{~cm}^{-3}\right)$ or dusty gas. The NGC $3393 \mathrm{Mg} \mathrm{II} / \mathrm{H} \beta$ ratio is also consistent with the dusty simulations. HST observations of the narrow lines of several high-luminosity AGNs (Wills et al. 1993) also yield ratios that are more consistent with the dusty simulations. Clearly, uniform, long-slit, high signalto-noise, UV to infrared spectra are required to understand the narrow emission line spectra of AGNs. Future observations using the Space Telescope Imaging Spectrograph (STIS) on HST will accomplish a good part of this for the first time.

\subsubsection{Optical Lines}

The predicted narrow optical forbidden lines are generally within the observed ranges of the mean spectrum, with the exceptions of $[\mathrm{Ne} \mathrm{III}]$ and $[\mathrm{Ne} \mathrm{v}]$, both being slightly 
TABLE 1

OBSERVED VERSUS INTEGRATED SPECTRA

\begin{tabular}{|c|c|c|c|c|c|c|}
\hline $\begin{array}{l}\text { Line } \\
(1)\end{array}$ & $\begin{array}{c}\text { Mean Seyfert } 2^{a} \\
\text { (2) }\end{array}$ & $\begin{array}{l}\text { Reference }^{\mathrm{b}} \\
\text { (3) }\end{array}$ & $\begin{array}{l}\text { NGC } 3393^{\circ} \\
\text { (4) }\end{array}$ & $\begin{array}{l}\text { Circinus }^{d} \\
\text { (5) }\end{array}$ & $\begin{array}{l}\text { Solar }^{\mathrm{e}} \\
\text { (6) }\end{array}$ & $\begin{array}{l}\text { Dusty }^{f} \\
\text { (7) }\end{array}$ \\
\hline$W_{\lambda}(\mathrm{H} \beta)^{\mathrm{g}}$ & $5-30$ & $\mathrm{~N}$ & $\ldots$ & $\ldots$ & 152 & 61 \\
\hline$W_{\lambda}(\mathrm{H} \beta)^{\mathrm{g}}$ & $15-100$ & BFP & $\cdots$ & $\cdots$ & $\ldots$ & $\cdots$ \\
\hline $\mathrm{L} \alpha \lambda 1216 \ldots$ & $55 \pm 20$ & FO & 20.9 & $\ldots$ & 28.7 & 9.62 \\
\hline $\mathrm{N} v \lambda 1240$ & $\ldots$ & $\ldots$ & 1.42 & $\ldots$ & 0.43 & 0.60 \\
\hline Si IV $\lambda 1397$ & $\ldots$ & $\ldots$ & $<0.58$ & $\ldots$ & 0.09 & 0.03 \\
\hline O Iv] $\lambda 1402 \ldots \ldots \ldots \ldots$ & $\ldots$ & & $<0.84$ & $\ldots$ & 0.22 & 0.45 \\
\hline $\mathrm{C}$ iv $\lambda 1549 \ldots \ldots \ldots \ldots$ & $12 \pm 8$ & FO & 4.75 & $\ldots$ & 3.70 & 2.47 \\
\hline He II $\lambda 1640 \ldots \ldots \ldots \ldots$ & - & & 1.75 & $\ldots$ & 2.18 & 2.82 \\
\hline C III $\lambda 1909 \ldots \ldots \ldots \ldots \ldots$ & $5.5 \pm 3.7$ & FO & 1.60 & $\ldots$ & 1.25 & 3.63 \\
\hline $\mathrm{C}$ II $\lambda 2326 \ldots \ldots \ldots \ldots \ldots$ & $\ldots$ & $\ldots$ & 0.25 & $\ldots$ & 0.39 & 0.97 \\
\hline$[\mathrm{Ne}$ IV $] \lambda 2424 \ldots$ & $\ldots$ & $\ldots$ & 0.32 & $\ldots$ & 0.19 & 0.31 \\
\hline$[\mathrm{O}$ II] $\lambda 2471 \ldots \ldots \ldots \ldots$ & $\ldots$ & $\ldots$ & 0.11 & $\ldots$ & 0.16 & 0.17 \\
\hline Mg II $\lambda 2798 \ldots \ldots \ldots \ldots \ldots$ & $1.8 \pm 1.5$ & FO & 0.43 & $\ldots$ & 1.42 & 0.23 \\
\hline He II $\lambda 3205 \ldots \ldots \ldots \ldots \ldots$ & & & 0.13 & $\ldots$ & 0.12 & 0.15 \\
\hline$[\mathrm{Ne} \mathrm{v}] \lambda 3426 \ldots \ldots \ldots \ldots$ & $1.2 \pm 0.2$ & FO & 0.36 & $\ldots$ & 0.62 & 0.67 \\
\hline$[\mathrm{O}$ II $] \lambda 3727 \ldots \ldots \ldots \ldots$ & $3.2 \pm 1.7$ & FO & 2.27 & $\ldots$ & 2.32 & 3.20 \\
\hline$[\mathrm{Ne}$ III $] \lambda 3869 \ldots \ldots \ldots \ldots$ & $1.4 \pm 0.4$ & FO & 0.81 & $\ldots$ & 1.13 & 1.00 \\
\hline$[\mathrm{S}$ II] $\vec{\lambda} 4074 \ldots \ldots \ldots \ldots$ & $0.3 \pm 0.2$ & FO & 0.12 & $\ldots$ & 0.26 & 0.28 \\
\hline $\mathrm{H} \delta \vec{\lambda} 4102 \ldots \ldots \ldots \ldots \ldots$ & $\ldots$ & $\ldots$ & 0.18 & $\ldots$ & 0.27 & 0.27 \\
\hline $\mathbf{H} \gamma \quad \lambda 4340 \quad \ldots \ldots \ldots \ldots \ldots$ & $\ldots$ & $\ldots$ & 0.48 & $\ldots$ & 0.48 & 0.47 \\
\hline$[\mathrm{O}$ III $] \lambda 4363 \ldots \ldots \ldots \ldots$ & $0.2 \pm 0.1$ & FO & 0.09 & - & 0.21 & 0.22 \\
\hline He II $\lambda 4686 \ldots \ldots \ldots \ldots \ldots$ & $0.3 \pm 0.1$ & FO & 0.25 & 0.30 & 0.30 & 0.37 \\
\hline $\mathrm{H} \beta \lambda 24861 \ldots \ldots \ldots \ldots \ldots$ & $1.00^{-}$ & FO & 1.00 & 1.00 & 1.00 & 1.00 \\
\hline$[\mathrm{O}$ III $] \lambda 5007 \ldots \ldots$ & $10.8 \pm 3.0$ & FO & 10.2 & 11.8 & 11.7 & 10.8 \\
\hline$\left[\mathrm{N}_{\mathrm{I}}\right] \lambda 5199 \ldots \ldots \ldots \ldots . .$. & $0.15 \pm 0.09$ & FO & 0.15 & 0.35 & 0.07 & 0.28 \\
\hline$[\mathrm{Fe}$ xIV $] \lambda 5303 \ldots \ldots \ldots \ldots$ & & & & $<0.10$ & 0.033 & 0.004 \\
\hline He I $25876 \ldots \ldots \ldots \ldots \ldots$ & $0.13 \pm 0.06$ & FO & $<0.11$ & & 0.13 & 0.11 \\
\hline$[\mathrm{Fe} \mathrm{VII}] \lambda 6087 \ldots \ldots \ldots \ldots$ & $0.10 \pm 0.05$ & FO & $\ldots$ & 0.055 & 0.049 & 0.009 \\
\hline$\left[\mathrm{O}_{\mathrm{I}}\right] \lambda 6300 \ldots \ldots \ldots \ldots \ldots$ & $0.57 \pm 0.20$ & FO & 0.35 & 0.36 & 0.56 & 0.84 \\
\hline$[\mathrm{Fe} \mathrm{x}] \lambda 6374 \ldots \ldots \ldots \ldots$ & $0.04 \pm 0.04$ & FO & $\ldots$ & 0.010 & 0.015 & 0.002 \\
\hline $\mathrm{H} \alpha \lambda 6563 \ldots \ldots \ldots \ldots \ldots$ & $3.1 \pm 0.1$ & FO & 3.00 & 2.73 & 2.96 & 2.91 \\
\hline$[\mathrm{N}$ II $] \lambda 6584 \ldots \ldots \ldots \ldots$ & $2.9 \pm 1.0$ & FO & 3.64 & 3.19 & 1.18 & 1.69 \\
\hline$[\mathrm{S}$ II $] \lambda 6720 \ldots \ldots \ldots \ldots \ldots$ & $1.5 \pm 0.5$ & FO & $\ldots$ & 1.64 & 1.20 & 1.87 \\
\hline$[\mathrm{Ar}$ III $] \quad \lambda 7135 \ldots \ldots \ldots \ldots$ & $0.24 \pm 0.07$ & FO & $\ldots$ & 0.16 & 0.22 & 0.27 \\
\hline$\left[\begin{array}{ll}\mathrm{Ca} & \mathrm{II}\end{array}\right] \lambda 7291 \ldots \ldots \ldots \ldots$ & $0.04 \pm 0.04$ & VB & $\ldots$ & .. & 0.16 & 0.002 \\
\hline$[\mathrm{O}$ II $] \lambda 7325 \ldots \ldots \ldots \ldots \ldots$ & $0.10-0.46^{\mathrm{h}}$ & DS & $\ldots$ & 0.073 & 0.20 & 0.21 \\
\hline$[\mathrm{Fe} \mathrm{xI}] \lambda 7892 \ldots \ldots \ldots \ldots$ & & & $\ldots$ & 0.066 & 0.068 & 0.008 \\
\hline$[\mathrm{S}$ III $] \lambda \lambda 9069,9532 \ldots \ldots$ & $0.5-4.5^{\mathrm{h}}$ & DS & $\ldots$ & 0.94 & 1.40 & 1.61 \\
\hline$[\mathrm{S}$ vIII $] \lambda 9915 \ldots \ldots \ldots \ldots$ & $\ldots$ & $\ldots$ & $\ldots$ & 0.044 & 0.024 & 0.022 \\
\hline$[\mathrm{S} \mathrm{Ix}] 1.252 \mu \mathrm{m} \ldots \ldots \ldots \ldots$ & $\ldots$ & & $\ldots$ & 0.042 & 0.11 & 0.086 \\
\hline$[\mathrm{Fe}$ II $] 1.257 \mu \mathrm{m} \ldots \ldots \ldots$ & $0.12 \pm 0.01$ & $\mathrm{~T}$ & $\ldots$ & 0.09 & 1.16 & 0.23 \\
\hline$[\mathrm{Fe} \mathrm{II}] 1.279 \mu \mathrm{m} \ldots \ldots \ldots$ & $0.03 \pm 0.01$ & $\mathrm{~T}$ & $\ldots$ & 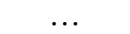 & 0.05 & 0.01 \\
\hline $\mathrm{P} \beta 1.2818 \mu \mathrm{m} \ldots \ldots \ldots \ldots$ & $0.16 \pm 0.01^{\mathrm{i}}$ & $\mathrm{T}$ & $\ldots$ & 0.15 & 0.16 & 0.15 \\
\hline$[\mathrm{Fe} \mathrm{II}] 1.534 \mu \mathrm{m} \ldots \ldots \ldots$ & $<0.02 \pm 0.01$ & $\mathrm{~T}$ & $\ldots$ & $\ldots$ & 0.10 & 0.01 \\
\hline$[\mathrm{Fe} \mathrm{II}] 1.644 \mu \mathrm{m} \ldots \ldots \ldots$ & $0.09 \pm 0.01$ & $\mathrm{~T}$ & $\ldots$ & 0.07 & 1.12 & 0.23 \\
\hline$[\mathrm{Fe}$ II $] 1.677 \mu \mathrm{m} \ldots \ldots \ldots$ & $0.02 \pm 0.01$ & $\mathrm{~T}$ & $\ldots$ & $\ldots$ & 0.07 & 0.01 \\
\hline$[\mathrm{Si} \mathrm{vI}] 1.96 \mu \mathrm{m} \ldots \ldots \ldots . .$. & $\ldots$ & $\ldots$ & $\ldots$ & 0.088 & 0.088 & 0.015 \\
\hline $\mathrm{Br} \gamma 2.166 \mu \mathrm{m} \ldots \ldots \ldots \ldots$ & $\ldots$ & $\ldots$ & $\ldots$ & $0.027^{\mathrm{i}}$ & 0.029 & 0.026 \\
\hline$[\mathrm{Ca}$ vIII $] 2.32 \mu \mathrm{m} \ldots \ldots \ldots$ & $\ldots$ & & $\ldots$ & 0.065 & 0.15 & 0.002 \\
\hline$[\mathrm{Si}$ VII $] 2.48 \mu \mathrm{m} \ldots \ldots \ldots$. & $\ldots$ & $\ldots$ & $\ldots$ & 0.15 & 0.11 & 0.020 \\
\hline$[\mathrm{Si} \mathrm{Ix}] 3.94 \mu \mathrm{m} \ldots \ldots \ldots .$. & $\ldots$ & $\ldots$ & $\ldots$ & 0.19 & 0.17 & 0.027 \\
\hline $\mathrm{Br} \alpha 4.052 \mu \mathrm{m} \ldots \ldots \ldots \ldots$ & $\ldots$ & $\ldots$ & $\ldots$ & $0.079^{\mathrm{i}}$ & 0.080 & 0.073 \\
\hline$[\mathrm{Fe} \mathrm{II}] 25.98 \mu \mathrm{m} \ldots \ldots \ldots$ & $\ldots$ & $\ldots$ & $\ldots$ & $\ldots$ & 0.64 & 0.13 \\
\hline
\end{tabular}

${ }^{a}$ The range of observed values taken from the reference in col. (3).

${ }^{b}$ Reference of the observed values: N is Netzer 1990; BFP is Binette, Fosbury, \& Parker 1993; FO is Ferland \& Osterbrock 1986; VB is from Villar-Martín \& Binette 1997; DS denotes a range of values from Dopita \& Sutherland 1995; and T is from an observation of NGC 4151 by Thompson 1995.

${ }^{c}$ Observations of NGC 3393 from Cooke et al. 1997.

${ }^{\mathrm{d}}$ Observations of the Circinus galaxy from Oliva et al. 1994.

${ }^{\mathrm{e}}$ Predictions from the integrated spectrum assuming solar abundances indicated by the square in Fig. 3 .

${ }^{\mathrm{f}}$ Predictions from the integrated spectrum assuming $\mathrm{H}$ II region abundances indicated by the star in Fig. 3.

${ }^{g}$ Equivalent width of $\mathrm{H} \beta$ in $\AA$.

${ }^{\mathrm{h}}$ These observations are given as a range.

${ }^{i}$ Assuming case B conditions.

underpredicted by $\sim 50 \%$ and $\sim 20 \%$, respectively, indicating possible differences in abundances. However, these lines are predicted to be stronger relative to $\mathrm{H} \beta$ than observed in NGC 3393. The [S II], [S III] lines are within the observed ranges of the mean Seyfert 2 spectrum.

Both integrations of the predicted nitrogen line $[\mathrm{N} \mathrm{I}]$ are consistent with the observed mean Seyfert 2 range, although the $[\mathrm{N} \mathrm{II}]$ line is predicted to be lower than observed by 
nearly half. Comparisons between both integrations and NGC 3393 of the nitrogen emission lines ([N I], [N II], and $\mathrm{N}$ v) relative to $\mathrm{H} \beta$ indicate that nitrogen may be enhanced in that object relative to the solar value by a factor of $\sim 2$.

The strength of the $\mathrm{He}$ II $\lambda 4686$ permitted line relative to $\mathrm{H} \beta$ is often used to constrain the ionizing continuum. A stronger $\mathrm{He}$ II $\lambda 4686$ line (relative to $\mathrm{H} \beta$ ) is indicative of more $\mathrm{He}^{+}$ionizing photons. Simple photoionization models often underpredict the line while doing well with the rest of the optical spectrum (Ferland \& Osterbrock 1986). Figure 3 and Table 1 show that the integrated model can predict this line within the observed range. Our fiducial integrations given in columns (6) and (7) of the table overpredict the entire $\mathrm{He}$ II spectrum relative to $\mathrm{H} \beta$ when compared with NGC 3393, which indicates that NGC 3393 has a slightly softer continuum than the one assumed here ( $\$ 2.1$ ).

The predictions of the relative strengths of the optical iron coronal lines, [Fe VII], [Fe X], [Fe XI], and [Fe XIV] to $\mathrm{H} \beta$ are in accord with the observations of the Circinus galaxy (Oliva et al. 1994) for the dust-free integrations. The dusty integrations underpredict these lines by factors of 5-10. However, Oliva et al. (1994), Morisset \& Péquignot (1996), and Ferguson, Korista, \& Ferland (1997) discuss the apparent large uncertainties in the collision strengths of [Fe x] $26375,[\mathrm{Fe} \mathrm{XI}]$ 27892, and [Fe XIV] 25303 . Given these uncertainties, we set the collision strengths for these transitions equal to 1 prior to running these simulations (note: Ferguson et al. used the collision strengths from the literature). These lines will not be useful probes of the highionization narrow-line region until this issue is resolved.

The weakness of the optical doublet [Ca II] $\lambda \lambda 7291,7324$ has been cited as evidence for the presence of dust grains with the NLR (Ferland 1993; Kingdon, Ferland, \& Feibelman 1995). Table 1 indicates that the computations assuming solar abundances overpredict the [Ca II] $\lambda 7291$ line by factors of 3-160, with the dusty calculations on the very low end of the observations. This suggests that calcium is actually depleted by factors up to 160 compared with its solar abundance.

\subsubsection{Infrared Lines}

The infrared forbidden [ $\mathrm{Fe}$ II] lines from the integrations described above are compared with the observations of NGC 4151 made by Thompson (1995). Because Thompson's observations included $\mathrm{P} \beta$, we can reference the [Fe II] to $\mathrm{H} \beta$, as in Table 1 , by assuming case $\mathrm{B}$ conditions for hydrogen. This is a valid assumption, since $\mathrm{P} \beta / \mathrm{H} \beta$ predicted by our integrations is very nearly the case $B$ ratio. Table 1 shows that the predicted intensities of the "dust-free" integration (col. [6]) are clearly too strong to match the observations, with some lines overpredicted by a factor of 10. Even for the "dusty" integration (col. [7]), where the iron abundance is depleted by a factor of 10 , relative to the solar value, the strongest $[\mathrm{Fe}$ II] lines are too strong by a factor of 2 compared with $\mathrm{H} \beta$. This evidence suggests that iron may be depleted by factors up to 20 in NGC 4151.

Simpson et al. (1996) and Veilleux, Goodrich, \& Hill (1997) present IR spectroscopy of a number of Seyfert 2 galaxies. Simpson et al. found [Fe II] $1.257 \mu \mathrm{m} / \mathrm{P} \beta \approx 1.21$ $(\sigma \approx 0.81)$, and Veilleux et al. found [Fe II] $1.257 \mu \mathrm{m} /$ $\mathrm{P} \beta \approx 1.24(\sigma \approx 0.90)$. As in NGC 4151 , these observed values are better matched to the predicted ratio from the dusty integration in Table $1(\approx 1.4)$. The dust-free integration predicts a ratio of $\approx 7$, much like the ratios arising in the shocked (grain free) environments of supernova remnants, plotted by both Simpson et al. (1996) and Veilleux et al. (1997).

Although the $[\mathrm{Fe}$ II] line ratios are too strong compared with the hydrogen lines, indicating an abundance difference, the relative $[\mathrm{Fe}$ II] spectrum is fairly consistent with the observations. Thompson (1995) discusses at length the probable difficulties with the observation of weaker lines, such as $1.279 \mu \mathrm{m}$ and $1.677 \mu \mathrm{m}$.

Table 1 also includes predictions of the relative strength of the far-IR [Fe II] $25.98 \mu \mathrm{m}$ line, the lowest transition within the ${ }^{6} \mathrm{D}$ ground term. This line is predicted to be quite strong in the solar integration (4 times $\mathrm{P} \beta$ ). Recall that the simulations presented here excluded gas with $T_{e}<3000 \mathrm{~K}$, and emission from these low-excitation lines might still be important at lower electron temperatures. In fact, we find that this line and others that arise within the ground term will have substantial contributions from AGN-heated photodissociation regions, if present. None of the $\sim 1 \mu \mathrm{m}$ $[\mathrm{Fe} \mathrm{II}]$ lines are emissive in gas temperatures below $3000 \mathrm{~K}$.

Our simulations of the infrared coronal lines shown in Table 1 are generally in accord with the observations of the Circinus galaxy for the dust-free integration. The [Si vI], [Si VII], and [Si IX] spectrum is well matched by the fiducial dust-free integrations. The dusty models do a poor job at matching the observations, particularly the [Ca VIII] line. Our dust-free fiducial model predicts this line to within a factor of 2, whereas the dusty integration underpredicts this line by over a factor of 30 . The predicted strength of the optical coronal line $[\mathrm{Fe} \mathrm{VII}] \lambda 6087$ is also consistent with the proposition that the high-ionization lines are emitted in a dust-free environment.

\section{LINE WIDTH AND CRITICAL DENSITY}

Filippenko \& Halpern (1984) showed that the line width of optical forbidden lines is correlated with the critical density of those lines. Other observers (Filippenko 1985; Appenzeller \& Östereicher 1988; Espey et al. 1994; Ho, Filippenko, \& Sargent 1996) have extended this work. It was realized by Filippenko \& Halpern that the probable explanation for the observed correlation is that the lines emit most efficiently near their critical densities and that the line emission is spatially stratified. Our LOC models are a quantitative formulation of this idea. By just glancing at Figure 1, one observes that the peak emission of the set of lines shown occurs over a range of gas density and distance. If the dominant velocity field is one that diminishes with distance from the nucleus, then we expect that those lines that are emitted optimally at smaller distances [larger $n(\mathrm{H})$ ] will have broader line widths than those that are optimally emitted farther away [smaller $n(\mathrm{H})$ ] and qualitatively agree with the observed correlation. Consider the contour plots of [O III] $\lambda 4363$ and [S II] $\lambda 6720$ shown in Figure 1. It is clear by looking at their places of peak emission that [O III] will be much broader than [S II], as is observed.

Figure 4 shows the results of a simple calculation of relative line widths assuming that the density-distance phasespace system of clouds obey the covering fraction and density indices of the fiducial square shown in Figure 3. In lieu of a more realistic velocity field that takes into account the mass distribution of the bulge in addition to the supermassive black hole, we assume virial velocities about a simple point mass of $10^{9} M_{\odot}$. In the figure we show the full width at half maximum (FWHM) and the full width at $10 \%$ 


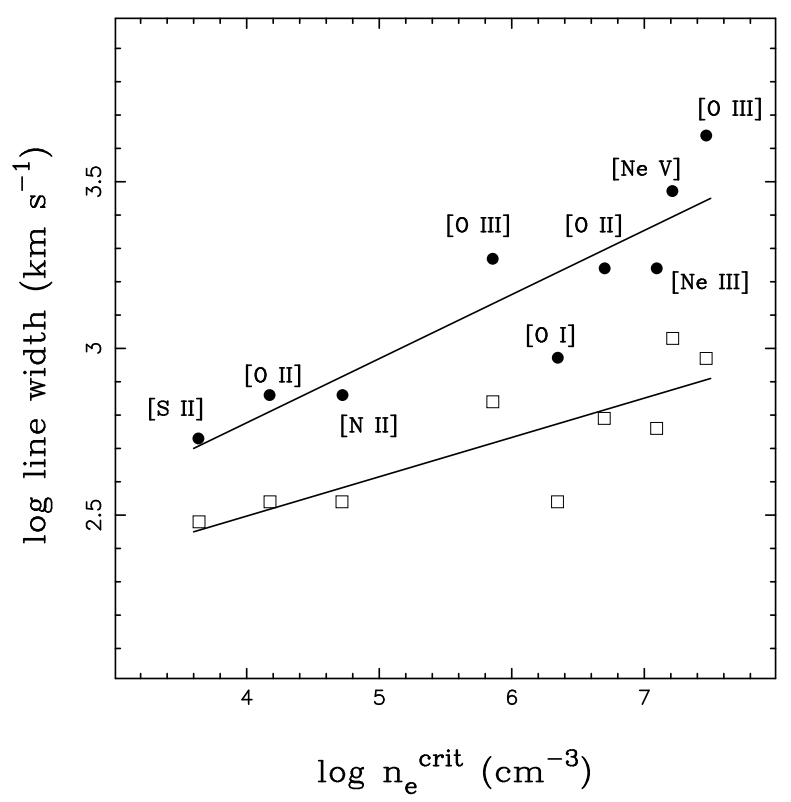

FIG. 4.- - Line width versus critical density of the square point shown in Fig. 3 . The solid circles are the full width at $10 \%$ intensity with a fitted slope of 0.193 . The open squares are FWHM with a fitted slope of 0.118 . The observed range in the slope is from 0.095 to 0.200 .

intensity (FW10\%) for the ions indicated versus the logarithm of the critical density $\left(n_{e}^{\text {crit }}\right)$. The slopes of the fitted linear regression in the figure were computed to be 0.193 (FW10\%) and 0.118 (FWHM), similar to those observed by Filippenko \& Halpern (1984) and Espey at al. (1994), which range from 0.095 to 0.200 .

\section{APPLICATION TO RESOLVED NLRS}

In a number of nearby Seyfert galaxies, the NLR is spatially resolved even on ground-based long-slit and imaging Fabry-Perot spectra. These NLRs frequently have the form either of ionization cones (cf. Pogge 1988; Tadhunter \& Tsvetanov 1989; Wilson et al. 1993; Robinson et al. 1994) or of apparent bow shocks and cocoon shocks around linear radio sources (Bower et al. 1995; Pogge \& De Robertis 1995; Capetti et al. 1996; Cooke et al. 1997). Typical scales are $200-400 h^{-1} \mathrm{pc} \mathrm{arcsec}^{-1}$, where $h=H_{0} / 100 \mathrm{~km} \mathrm{~s}^{-1}$ $\mathrm{Mpc}^{-1}$. With the imminent commissioning of the HST STIS spectrograph and ground-based adaptive optics systems, it should be possible to study spectroscopically a reasonable sample of NLRs with $\sim 20-40 \mathrm{pc}\left(\sim 10^{20} \mathrm{~cm}\right)$ resolution.

The exact scaling of such dimensions onto the $y$-axis of Figures 1 and 2 is complicated by the fact that the (probably beamed) ionizing continuum luminosity seen by these NLRs is not directly measured. However, simple arguments based on counting recombination photons (cf. Binette, Fosbury, \& Parker 1993) imply that the gas sees an ionizing luminosity that is, on average, a factor of 10 larger than what we see. Therefore, the range in incident flux used in the model grid presented here is applicable to these resolved NLRs, and it is of interest to consider the predicted radial dependence of various line intensity ratios when integrated over the cloud gas density distribution.

Figure 5 shows four different line ratios indicating the gas density, temperature, and ionization as functions of distance and gas density in the form of logarithmic contour plots.
The figure also plots the ratios of the line intensities integrated over clouds of all densities, as functions of distance from the ionizing source; shown are the results of two different integrations along the density axis.

\subsection{Density}

The density indicator [ $\mathrm{S} \mathrm{II]} \lambda 6716 / \lambda 6731$ is shown in the top two panels of Figure $5 a$. The line ratio has the linear value of 1.36 in the extreme upper left-hand corner of the plot at $[\log n(\mathrm{H})=2.0, \log R=22.0]$. Moving down and to the right along a line of constant ionization parameter, the solid lines are 1 dex increments, and the dashed lines are 0.1 dex steps. The large flat plain of constant ratio corresponds to the high-density limit or a linear value of $\sim 0.44$.

The line ratio as a function of distance shows that for the integrations presented, the high-density limit is predicted at small distances. The solid line [integrated assuming that $\left.g(n) \propto n^{-1.0}\right]$ remains at that limit for larger distances, whereas the dashed line [integrated assuming a steeper density distribution, i.e., $\left.g(n) \propto n^{-1.8}\right]$ turns upward sooner. The dashed curve reaches a peak value nearly equal to the low-density limit given by Osterbrock (1989). The gas density inferred from the dashed curve falls off as $R^{-2 / 3}$, while the density inferred from the solid curve is pinned at the high-density limit for larger values of $R$, then falls off more steeply than $R^{-2 / 3}$.

\subsection{Temperature}

The temperature indicator [O III] $\lambda 5007 / \lambda 4363$ (note that $\lambda 4959$ is not included) is shown in the bottom two plots of Figure $5 a$. The contour plot has a peak value of 112 in the upper left-hand corner $[\log n(\mathrm{H})=2.0, \log R=21.0)$. Following a line of constant ionization parameter down and to the right, the solid contour line (which has a backward " $S$ " shape) has the logarithmic value of 2.0, and the dashed lines are 0.2 dex increments. The outer contour in the lower right-hand part of the plot has a logarithmic value of -1.0 at $[\log n(\mathrm{H})=10.0, \log R=18.0]$. The contour plot shows that in the low-density limit $\left[\log n(\mathrm{H}) \lesssim 4.5 \mathrm{~cm}^{-3}\right]$, the ratio is a good temperature indicator in that moving perpendicular to the ionization parameter, the ratio changes are roughly independent of density. At higher densities, the ratio becomes dependent on both temperature and density.

The gas density integrated line ratio as a function of distance shows that for large distances from the ionizing source, the ratio is independent of the weighting of the density axis; i.e., the ratio is in the low-density limit. Using the analytical expression from Osterbrock (1989), the ratio can be converted into temperature in the low-density limit. For the range $20.0 \mathrm{~cm} \leq \log R \leq 22.0 \mathrm{~cm}$, the inferred temperature has the range $14,000 \mathrm{~K} \geq T_{e} \geq 8400 \mathrm{~K}$, falling off very gradually as approximately $R^{-1 / 9}$. At smaller radii, the $\lambda 5007 / \lambda 4363$ ratio further decreases, reflecting, mainly, the drop in $\lambda 5007$ due to collisional quenching and the increase of $\lambda 4363$, which continues to emit efficiently up to its critical density, rather than reflecting an increase in temperature.

The $[\mathrm{N}$ II] $\lambda 5755 / \lambda 6584$ line ratio is also a useful temperature indicator. Wilson, Binette, \& Storchi-Bergmann (1997) use this ratio in concert with the [O III] $\lambda 5007 / \lambda 4363$ ratio as evidence for the presence of matter-bounded clouds in the NLR. Defining $R_{[\mathrm{N} \mathrm{II]}}$ as the [N II] line ratio and $R_{\text {[O III] }}$ as the [O III] line ratio, our model integrations predict that $R_{[\mathrm{O} \text { III] }}$ versus $R_{[\mathrm{N} \mathrm{II]}}$ depends little on the density weighting factor $g(n)$. We find a linear-log relationship of 


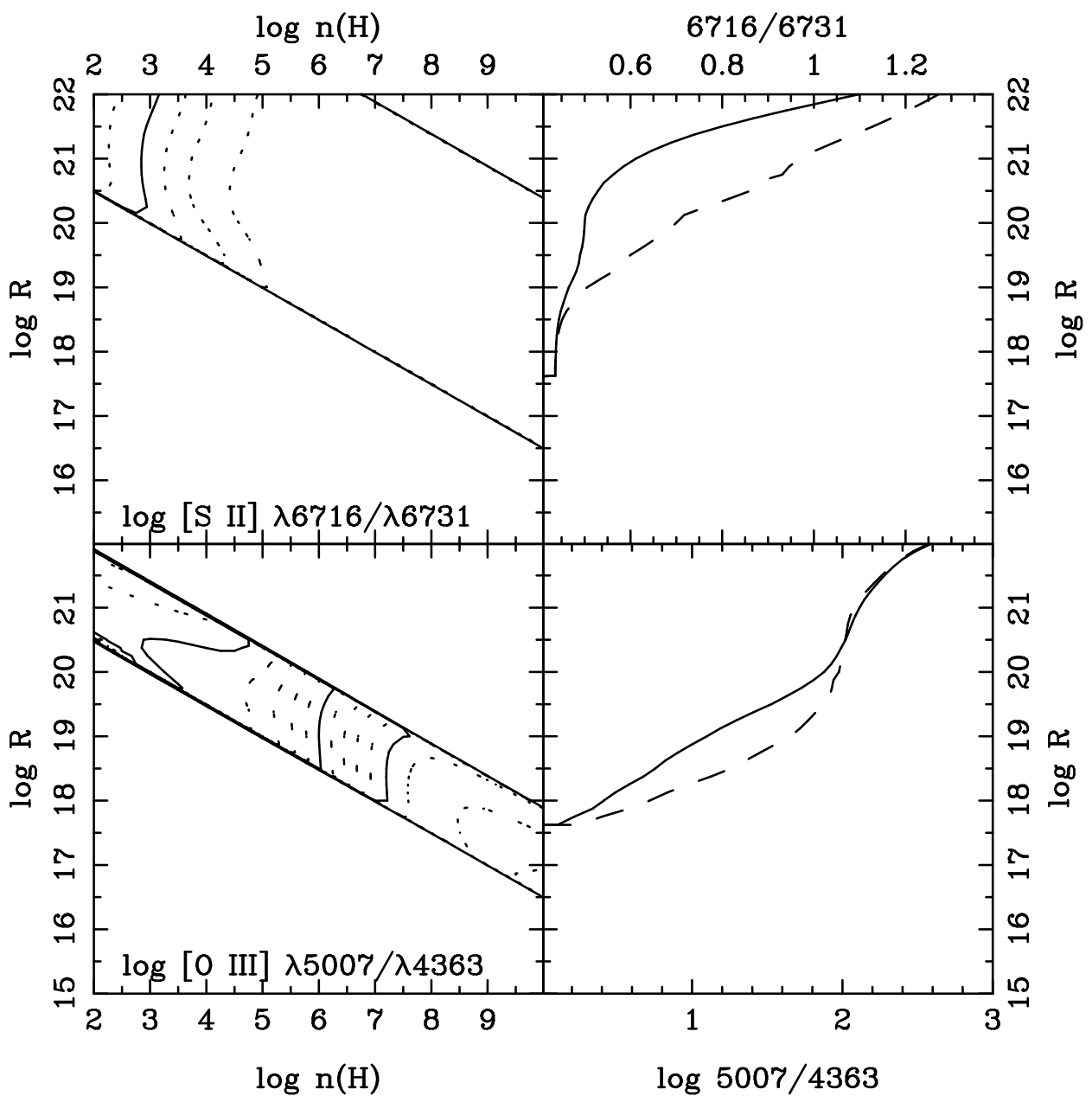

FIG. $5 a$

Fig. 5.-Line ratio diagnostic diagrams of gas density, temperature, and ionization shown in two different forms. The left-hand column of plots in the figure are logarithmic contour diagrams of the ratios indicated taken from the simulations shown in Fig. 1. The right-hand column of plots in the figure are graphs of the indicated line ratio versus distance from the central source for two different integrations as discussed in $\S 3$, but integrated in the density dimension only. The solid lines are integrations assuming that $g(n) \propto n^{-1.0}$, and the dashed lines assume $g(n) \propto n^{-1.8}$. Each line ratio is shown on a different scale; we will discuss each in turn in the text in $\S 5$.

the form

$$
\log R_{[\mathrm{O} \mathrm{III}]}=0.1593 \log R_{[\mathrm{N} \mathrm{II}]}+0.1932 .
$$

The fiducial dust-free integration from Figure 3 (§ 3.1) predicts $R_{[\mathrm{O} I \mathrm{II}]}=0.0182$ and $R_{[\mathrm{N} \mathrm{II]}}=0.0215$, and the fiducial dusty simulation predicts $R_{[\mathrm{O} \mathrm{III]}}=0.0206$ and $R_{[\mathrm{N} \mathrm{II]}}=$ 0.0229 . These correspond to temperature differences ( $T_{[\mathrm{O} \text { III] }}$ $\left.-T_{[\mathrm{N} \mathrm{III}}\right)$ of $3000 \mathrm{~K}$ and $3500 \mathrm{~K}$, respectively. These predictions lie in the range of observed values (Wilson, Binette, \& Storchi-Bergmann 1997) and do not require the presence of a significant population of matter-bounded clouds, although we do not exclude this possibility.

\subsection{Ionization}

The ratio [O III] $\lambda 5007 /(\mathrm{H} \alpha+[\mathrm{N}$ II $]$ 26584) has been used as an ionization indicator, and this ratio is shown in the top left-hand panel of Figure $5 b$. The outer contour has the linear value of 1.0 and the dashed lines are $+0.2 \mathrm{dex}$ steps. We have chosen to include both $\mathrm{H} \alpha$ and [N II], since many filters include both lines. The contour plot of this ratio is relatively flat over a wide range in density, distance, and ionization parameter. In fact, for a constant density of
$n(\mathrm{H})=10^{4} \mathrm{~cm}^{-3}$, the $[\mathrm{O} \mathrm{III}] /(\mathrm{H} \alpha+[\mathrm{N} \mathrm{III}])$ ratio would be constant from $\sim 10-50 L_{43.5}^{1 / 2} \mathrm{pc}$, even though the ionization parameter decreases by $\sim 10^{1.5}$ over the same range. A ratio that behaves in similar manner is [O $\mathrm{III}] \lambda 5007 / \mathrm{H} \beta$; it is shown in the bottom left-hand panel of Figure $5 b$. The outer contour has the linear value of 1.0 , and the dashed lines are +0.2 dex steps. The $[\mathrm{O} \mathrm{III}] / \mathrm{H} \beta$ ratio is just as flat over a wider range in ionization parameter, as is $[\mathrm{O} \mathrm{III}] /(\mathrm{H} \alpha+$ $[\mathrm{N} \mathrm{II}])$. Thus, while these line ratios are useful in separating $\mathrm{H}$ II region galaxies from active galaxies (cf. BPT), they are not reliable ionization parameter indicators.

In the top right-hand panel of Figure $5 b$, the [O III] $\lambda 5007 /(\mathrm{H} \alpha+[\mathrm{N} \mathrm{II}] 26584)$ ratio as a function of distance is shown. The ratio has the feature of being constant from $\sim 3-300 L_{43.5}^{1 / 2} \mathrm{pc}$ for the integration assuming a steep distribution $\left[g(n) \propto n^{-1.8}\right.$; dashed line $]$ of clouds along the density axis. The solid line $\left[g(n) \propto n^{-1.0}\right]$ is flat over a much smaller distance range, because in this integration emission from higher density gas is being included. The sharp falloff of the ratio at large distances $\left(R \geq 300 L_{43.5}^{1 / 2}\right.$ pc) occurs because the [O III] $\lambda 5007$ emissivity is dropping very quickly with distance at the lowest gas density $\left(10^{2} \mathrm{~cm}^{-3}\right)$ considered here, since the gas is underionized and cannot 


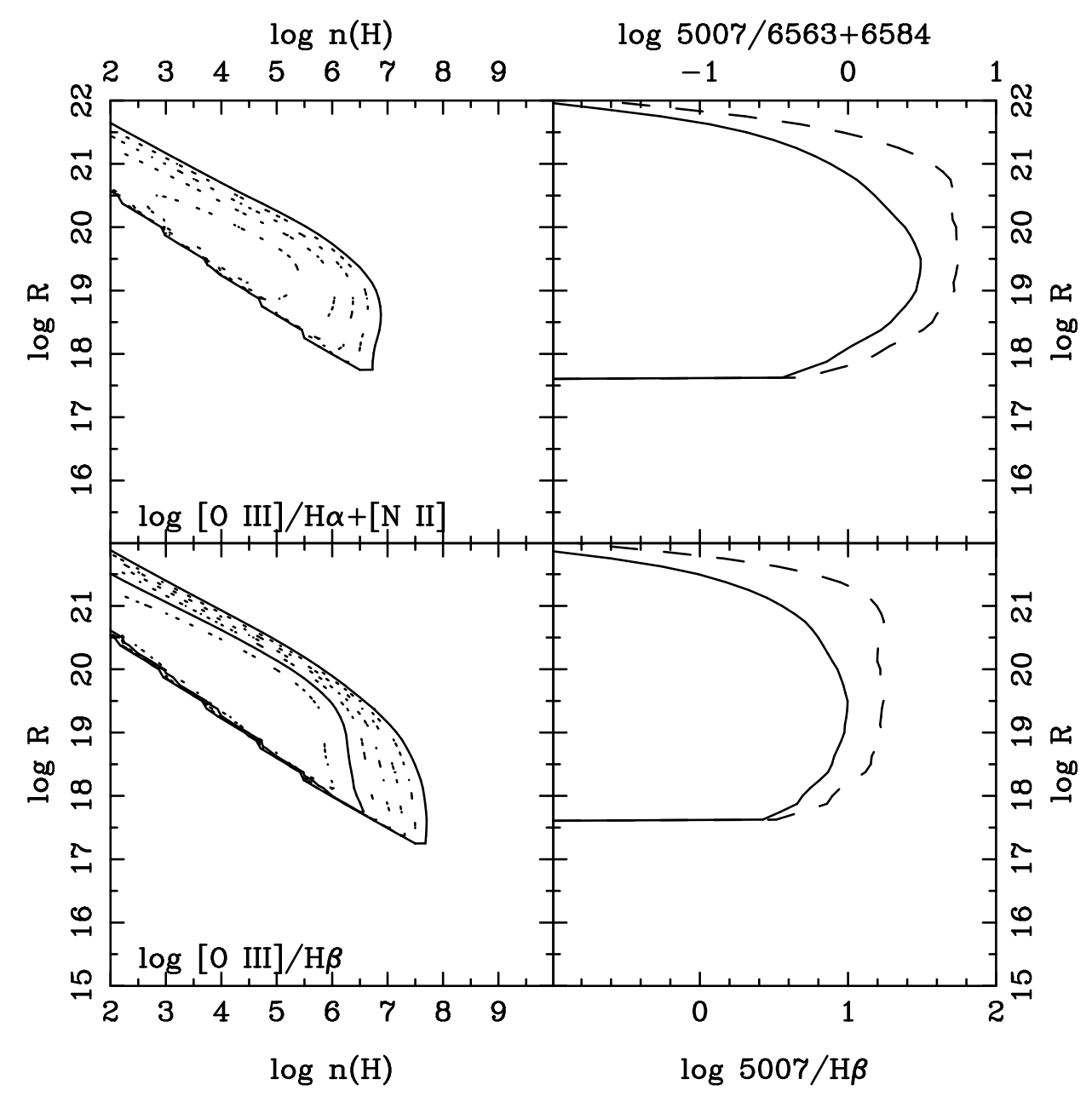

FIG. $5 b$

FIG. 5-Continued

produce the line efficiently, while the $[\mathrm{N}$ II] $\lambda 6584$ line is simultaneously increasing in strength (see Fig. 1). The bottom right-hand panel of Figure $5 b$ shows the integrated $[\mathrm{O} \mathrm{III]}] / \mathrm{H} \beta$ ratio as a function of distance; this ratio behaves similarly to the $[\mathrm{O} \mathrm{III]} /(\mathrm{H} \alpha+[\mathrm{N} \mathrm{II}])$ ratio. Both of these line ratios are observed to be relatively constant over factors of 3-4 in distance in several Seyfert 2 galaxies (Robinson et al. 1994; Capetti et al. 1996).

Figure $6 a$ shows the [O III] $\lambda 5007 /[\mathrm{O}$ II] $\lambda 3727$ line ratio as a contour plot. In the figure, the outer contour has a linear value of 1.0 , and the dashed lines are +0.2 dex steps. At low densities, the contours are closely spaced and parallel with ionization parameter, indicating that this line ratio is a good ionization indicator. At densities of $\sim 10^{4.5} \mathrm{~cm}^{-3}$, [O II] becomes collisionally quenched, while [O III] does not, thus the ratio becomes dependent on density as well.

In Figure $6 b$, we show the predicted [O III]/[O II] line ratio as a function of radius for four models compared with the observations of Robinson et al. (1994) for NGC 4151. The data have been placed on the distance axis by assuming that $1.0 \approx 100 \mathrm{pc}$, as used by Robinson et al. The model integrations have been rescaled in distance by factors of 4 , corresponding to a 16 -fold increase in the ionizing luminosity incident on the clouds. The observed strengths of these and a number of other emission lines in NGC 4151 suggest that the ionizing luminosity incident on the narrow- line clouds may be 10-20 times the observed one (Penston et al. 1990; Robinson et al. 1994), though the magnitude of the photon deficit is very uncertain (see also Schulz \& Komossa 1993). Since the ionizing luminosity employed here happens to match that of NGC 4151 emitted toward Earth (Robinson et al.), our rescaling is consistent with the continuum beaming inferred from the observations. The predicted $[\mathrm{O} \mathrm{III}] /[\mathrm{O} \mathrm{II}]$ ratio shown in Figure $6 b$ did not include emission from matter-bounded clouds; including such clouds would tend to enhance the $[\mathrm{O} \mathrm{III}] /[\mathrm{O} \mathrm{II}]$ ratio where it is small in Figure $6 b$.

Robinson et al. (1994) include several other line ratios as functions of projected distance for NGC 4151. These include $[\mathrm{Ne} \mathrm{v}] /[\mathrm{Ne}$ III $]$ and $[\mathrm{O} \mathrm{III}] /[\mathrm{N}$ II $]$, which behave similarly to $[\mathrm{O} \mathrm{III}] /[\mathrm{O} \mathrm{II}]$, shown in Figure $6 b$. Also observed are $[\mathrm{N} \mathrm{II}] / \mathrm{H} \alpha,[\mathrm{O} \mathrm{I}] / \mathrm{H} \alpha,[\mathrm{O}$ II $] / \mathrm{H} \alpha$, and $[\mathrm{S} \mathrm{II}] / \mathrm{H} \alpha$. Our simulations qualitatively match the general trends in these line ratios.

\section{IS THE NLR DUSTY?}

The presence of dust in the NLR is a vital clue to the origin of the gas. For instance, if shocks are prevalent in the emitting region, then grains will not survive (Donahue \& Voit 1993). Both the dust-free and dusty simulations reproduce the average narrow emission line ratios shown in Figure 3. However, other lines do indicate substantial 


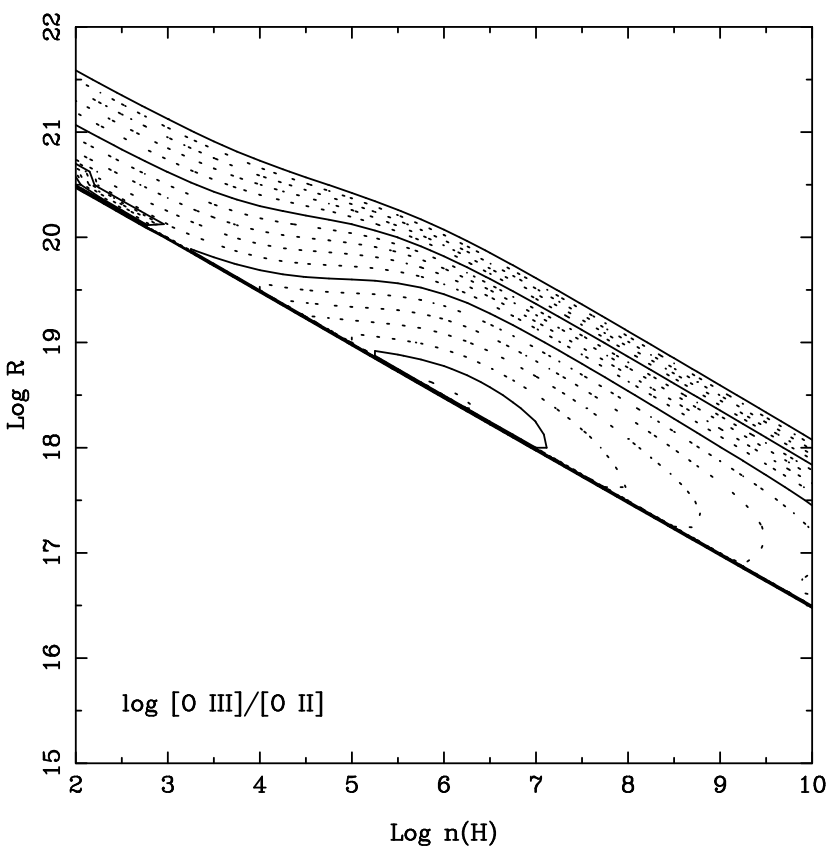

FIG. $6 a$

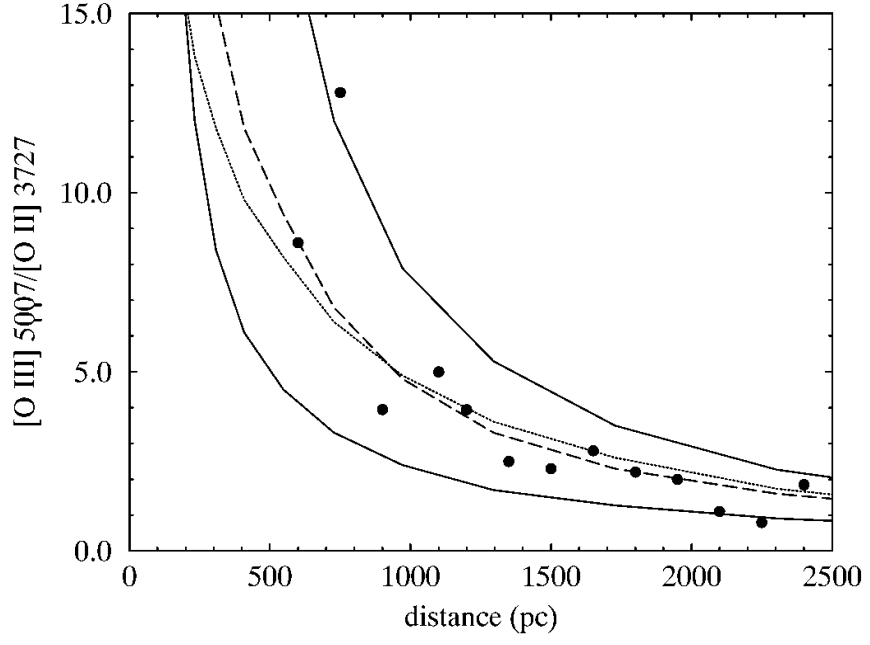

FIG. $6 b$

FIG. 6.-The predicted [O III] $\lambda 5007 /[\mathrm{O} \mathrm{II}] \lambda 3727$ ratio shown in two forms. (a) Logarithmic contour diagram of the ratio taken from the simulations shown in Fig. 1. The outer contour has a linear value of 1 , and the solid lines are 1.0 dex increments, with the dashed lines being 0.2 dex steps. $(b)$ The line ratio as function of distance from the ionizing source. The lower solid line is an integration in density assuming $g(n) \propto n^{-1.0}$, and the upper solid line assumes $g(n) \propto n^{-1.8}$. The dashed line is the fiducial integration with $g(n) \propto n^{-1.4}$ for the dust-free simulation. The dotted line is the fiducial integration with $g(n) \propto n^{-1.6}$ for the dusty simulation. All integrations have been rescaled by factors of 4 in distance, corresponding to 16 in luminosity (see $\S 5.3$ ). The filled circles are the observations of NGC 4151 by Robinson et al. (1994), assuming $1 . .0 \approx 100 \mathrm{pc}$.

depletions relative to the solar value. The absence of an observed [Ca II] $\lambda 7291$ line has led to speculation that the NLR is a dusty place (Ferland 1993; Kingdon, Ferland, \& Feibelman 1995). Recent observations of the [Ca II] line (Villar-Martín \& Binette 1997) show that the line is much weaker than the solar integrations predict (Table 1), supporting the conclusion of Villar-Martín \& Binette that $\mathrm{Ca}$ is sharply depleted. The simulations presented here indicate that $\mathrm{Ca}$ is depleted relative to the solar value by factors of 3-160 based on the range of observed line strengths when compared with the dust-free simulations in Table 1 . The results of the simulations for the infrared [Fe II] lines, as discussed in $\S 3.1 .3$, indicate that $\mathrm{Fe}$ is also underabundant relative to the solar value by factors of 10-20 in NGC 4151 . Comparisons of the compilations of the $[\mathrm{Fe}$ II] $1.257 \mu \mathrm{m} / \mathrm{P} \beta$ line ratio by Simpson et al. (1996) and Veilleux et al. (1997) with the simulations provide further evidence that the NLR is a dusty environment. A similar conclusion was reached by Simpson et al. (1996).

Two line ratios, $\mathrm{L} \alpha / \mathrm{H} \beta$ and $\mathrm{Mg} \mathrm{I} / \mathrm{H} \beta$, observed in NGC 3393 and discussed in $\S 3.1 .1$, also suggest that dust exists in the emitting gas. However, the uncertainty in the correction for the unintended positional error in the observations makes the conclusion from $\mathrm{L} \alpha / \mathrm{H} \beta$ uncertain. There is no positional error for the $\mathrm{Mg} \mathrm{II} / \mathrm{H} \beta$ ratio, and this ratio makes a stronger statement supporting the presence of dust in NGC 3393.

Evidence that a portion of the emitting gas is dust free is apparent in the observations of strong coronal lines in many AGNs. Oliva et al. (1994) have observed a very strong [Ca VIII $2.32 \mu \mathrm{m}$ infrared line and strong optical [Fe VII] 26087, [Fe x] 26374, [Fe XI] 27892, and [Fe XIV] $\lambda 5303$ lines, indicating that the gas is dust free. Simulations of coronal line emitting gas by Korista \& Ferland (1989), Oliva et al. (1994), Ferguson, Korista, \& Ferland (1997), and the results of those shown in Table 1 indicate that these elements are not depleted.

Taking the observations at face value, we are presented with a conundrum: the low-ionization UV Mg II, optical [Ca II], and infrared [Fe II] lines clearly indicate that dust is present, but the strong coronal lines suggest a dust-free environment. One possible reconciliation is to postulate that there is dust in the NLR, but only in the neutral or partially neutral portions of the clouds. In the simulations presented in this paper, we have assumed that the entire cloud is either dust free or dusty. It is not unreasonable to imagine that the highly ionized portion of NLR clouds to be dust free, perhaps because the grains have been exposed to a strong UV continuum for quite some time, and that in more neutral regions grains are free to exist. Another possibility is that high-ionization clouds have been processed through shocks, but those that emit more neutral ions have not. Both of these possibilities argue for a very complex NLR environment, where the emitting gas has a variety of origins.

\section{SUMMARY}

We have shown that the AGN narrow emission line spectra, from [O I] to [Si IX], can largely be reproduced by assuming that the emitting region consists of clouds with a wide range of gas densities and distances from the ionizing source. Here we chose simple power-law cloud distribution functions and the appropriate power-law indices $(\beta$ and $\gamma)$, whose resulting spectrum matched the observed [O III]/ $[\mathrm{O}$ II $]$ and $[\mathrm{O}$ III $] / \mathrm{H} \beta$ ratios. While smooth power-law distributions in cloud properties may be too simple, its success 
in predicting the observed spectrum points to the strength of the "locally optimally emitting cloud" scenario. The LOC model integrations result in the following:

1. The classical BPT line ratios for AGN narrow emission lines are reproduced (Fig. 3).

2. The predicted $\mathrm{H} \beta$ equivalent width, assuming full source coverage and an isotropic continuum, is more than sufficient to account for the observed emission in most Seyfert galaxies (Table 1). An anisotropic continuum will strengthen the predicted line equivalent widths and allow for smaller covering fractions. The predicted $[\mathrm{O}$ III $] /[\mathrm{O}$ II $]$ ratio as a function of radius implies that the narrow-line clouds see a continuum that is an order of magnitude more luminous than we observe in NGC 4151.

3. The LOC integration simultaneously reproduces the low [S II] $\lambda 6716 / \lambda 6731$ density and the high [O III] $\lambda 5007 /$ $\lambda 4363$ temperature (cf. Fig. $3 b$ ), resolving a long-standing problem.

4. The observed line width-critical density correlation for the optical forbidden lines can be reproduced. A natural consequence of an ensemble of clouds in the gas densitydistance plane is that lines with higher critical densities are formed at smaller distances (cf. Fig. 1). We have shown that if we assume that this ensemble of emitting clouds is placed in a gravitational potential, then the line width-critical density correlation very similar to that observed will naturally result.

5. The presence of a large density range at each radius can change the values of the classical density and ionization-level indicators to at least more closely mimic the observed radial gradients in these intensity ratios.

6. Low-ionization lines show clear evidence of depletion (Fig. 3 and Table 1), but high-ionization regions do not. It is possible that dust grains are selectively destroyed in highionization regions by shocks or sublimation and that the neutral regions are undisturbed. This argues for a variety of NLR cloud origins.

We thank Andrew Cooke for agreeing to our use of the NGC 3393 data. We acknowledge support from NASA and NSF through grants NAG-3223 and AST 93-19034, and STScI for GO-06006.02. We also thank the University of Kentucky Center for Computational Science and its director, John Connolly, for access to the Exemplar parallel supercomputer.
Acosta-Pulidó, J. A., Vila-Vilaró, B., Pérez-Fournon, I., Wilson, A. S., \& Tsvetanov, Z. I. 1996, ApJ, 464, 177

Appenzeller, I., \& Östreicher, R. 1988, AJ, 95, 45

Arribas, S., Mediavilla, E., \& García-Lorenzo, B. 1996, ApJ, 463, 509

Baldwin, J. A., et al. 1991, ApJ, 374, 580

.1996, ApJ, 468, L115

Baldwin, J. A., Ferland, G. J., Korista, K. T., \& Verner, D. A. 1995, ApJ, 455, L119

Baldwin, J. A., Phillips, M. M., \& Terlevich, R. 1981, PASP, 93, 5 (BPT)

Binette, L., Fosbury, R. A., \& Parker, D. 1993, PASP, 105, 1150

Binette, L., Wilson, A. S., \& Storchi-Bergmann, T. 1996, A\&A, 312, 365

Bower, G., Wilson, A., Morse, J. A., Gelderman, R., Whittle, M., \& Mulchaey, J. 1995, ApJ, 454, 106

Capetti, A., Axon, D. J., Macchetto, F., Sparks, W. B., \& Boksenberg, A. 1996, ApJ, 469, 554

Cooke, A. J., Baldwin, J. A., Ferland, G. J., Netzer, H., Wills, B. J., \&

Wilson, A. S. 1997, in preparation

Davidson, K. 1977, ApJ, 218, 20

Donahue, M., \& Voit, G. M. 1993, ApJ, 414, L17

Dopita, M. A., \& Sutherland, R. S. 1995, ApJ, 455, 468

Espey, B. R., et al. 1994, ApJ, 434, 484

Evans, I. N., Ford, H. C., Kinney, A. L., Antonucci, R. R. J., Armus, L., \& Caganoff, S. 1991, ApJ, 369, L27

Ferguson, J. W., Korista, K. T., \& Ferland, G. J. 1997, ApJS, 110, 287

Ferland, G. J. 1993, in The Nearest Active Galaxies, ed. J. Beckman, L. Colina, \& H. Netzer (Madrid: Consejo Superior de Investigaciones Cientificas), 75

. 1996, Hazy, a Brief Introduction to Cloudy, Univ. Kentucky Dept. Physics \& Astron. Internal Rep.

Ferland, G. J., \& Osterbrock, D. E. 1986, ApJ, 300, 658

Filippenko, A. V. 1985, ApJ, 289, 489

Filippenko, A. V., \& Halpern, J. P. 1984, ApJ, 285, 458

Falcke, H., Wilson, A. S., Simpson, C., \& Bower, G. A. 1996, 470, L31

Grevesse, N., \& Anders, E. 1989, in AIP Conf. Proc. 183, Cosmic Abundances of Matter, ed. C. J. Waddington (New York: AIP), 1

Grevesse, N., \& Noels, A. 1993, in Origin and Evolution of the Elements, ed. N. Prantzos, E. Vangioni-Flam, \& M. Casse (Cambridge: Cambridge Univ. Press), 15

Ho, L. C., Filippenko, A. V., \& Sargent, W. L. W. 1996, ApJ, 462, 183

Kingdon, J. B., Ferland, G. J., \& Feibelman, W. A. 1995, ApJ, 439, 793

Komossa, S., \& Schulz, H. 1997, A\&A, 323, 31

Korista, K. T., \& Ferland, G. J. 1995, ApJ, 343, 678

Laor, A., \& Draine, B. T. 1993, ApJ, 402, 441

Macchetto, F., Capetti, W. B., Sparks, W. B., Axon, D. J., \& Boksenberg, A. 1994, ApJ, 435, L15

Morisset, C., \& Péquignot, D. 1996, A\&A, 313, 611
Morse, J. A., Raymond, J. C., \& Wilson, A. S. 1996, PASP, 108, 426

Nelson, C. H., MacKenty, J. W., Simkin, S. M., \& Griffiths, R. E. 1996, ApJ, 466,713

Netzer, H. 1990, in Active Galactic Nuclei, ed. T. J. L. Courvoisier \& M. Mayor (SAAS-FEE Advanced Course 20, Swiss Society for Astrophysics and Astronomy; Berlin: Springer), 57

Netzer, H., \& Laor, A. 1993, ApJ, 404, L51

Oliva, E., Salvati, M., Moorwood, A. F. M., \& Marconi, A. 1994, A\&A, 288,457

Osterbrock, D. E. 1989, Astrophysics of Gaseous Nebulae and Active Galactic Nuclei (Mill Valley: University Science)

Osterbrock, D. E., Tran, H. D., \& Veilleux, S. 1992, ApJ, 389, 305

Penston, M. V., et al. 1995, A\&A, 236, 53

Pogge, R. W. 1988, ApJ, 328, 519

. 1997, in Emission Lines in Active Galaxies: New Methods and Techniques, ed. B. M. Peterson, F.-Z. Cheng, \& A. S. Wilson (San Francisco: ASP), in press

Pogge, R. W., \& De Robertis, M. M. 1995, ApJ, 451, 585

Pradhan, A. K., \& Zhang, H. L. 1993, ApJ, 409, L77

Quinet, P., Le Dourneuf, M., \& Zeippen, C. J. 1996, A\&AS, 120, 361

Robinson, A., et al. 1994, A\&A, 291, 351

Rubin, R. H., Erickson, E. F., Hass, M. R., Colgan, S. W. J., Simpson, J. P., \& Dufour, R. J. 1992, in IAU Symp. 150, The Astrochemistry of Cosmic Phenomena, ed. P. D. Singh (Dordrecht: Kluwer), 281

Rubin, R. H., Simpson, J. P., Hass, M. R., \& Erickson, E. F. 1991, ApJ, 374, 564

Schmitt, H. R., \& Kinney, A. L. 1996, ApJ, 463, 498

Schulz, H., \& Komossa, S. 1993, A\&A, 278, 29

Simpson, C., Forbes, D. A., Baker, A. C., \& Ward, M. J. 1996, MNRAS, 283,777

Tadhunter, C. N., \& Tsvetanov, Z. I. 1989, Nature, 341, 422

Thompson, R. I. 1995, ApJ, 445, 700

Tsvetanov, Z., \& Walsh, J. R. 1992, ApJ, 497, 485

Veilleux, S., Goodrich, R. W., \& Hill, G. J. 1997, ApJ, 477, 631

Verner, E., et al. 1997, in preparation

Villar-Martín, M., \& Binette, L. 1997, A\&A, 317, 350

Wills, B. J., Netzer, H., Brotherton, M. S., Han, M., Wills, D., Baldwin, J. A., Ferland, G. J., \& Browne, I. W. A. 1993, ApJ, 410, 534

Wilson, A. S. 1997, in Emission Lines in Active Galaxies: New Methods and Techniques, ed. B. M. Peterson, F.-Z. Cheng, \& A. S. Wilson (San Francisco: ASP), in press

Wilson, A. S., Binette, L., \& Storchi-Bergmann, T. 1997, ApJ, 482, 131

Wilson, A. S., Braatz, J. A., Heckman, T. M., Krolik, J. H., \& Miley, G. K. 1993, ApJ, 419, L61

Wood, B. E., \& Linsky, J. L. 1997, ApJ, 474, L39 